\title{
REDUCED DONALDSON-THOMAS INVARIANTS AND THE RING OF DUAL NUMBERS
}

\author{
GEORG OBERDIECK AND JUNLIANG SHEN
}

\begin{abstract}
Let $A$ be an abelian variety. We introduce $A$-equivariant Grothendieck rings and $A$-equivariant motivic Hall algebras, and endow them with natural integration maps to the ring of dual numbers. The construction allows a systematic treatment of reduced DonaldsonThomas invariants by Hall algebra techniques. We calculate reduced Donaldson-Thomas invariants for K3 $\times E$ and abelian threefolds for several imprimitive curve classes. This verifies (in special cases) multiple cover formulas conjectured by Oberdieck-Pandharipande and BryanOberdieck-Pandharipande-Yin.
\end{abstract}

\section{Contents}

1. Introduction

2. Multiple cover formulas

3. Equivariant Grothendieck rings

4. Equivariant motivic Hall algebras

5. Reduced DT invariants of $K 3 \times E$

6. Reduced DT invariants for abelian 3-folds

References

\section{INTRODUCTION}

1.1. Equivariant Hall algebras. We present a framework to apply techniques from motivic Hall algebras and Grothendieck rings of varieties in the presence of an action by an abelian variety. The idea is to incorporate the action as additional data into the definition, making the Hall algebra and the underlying Grothendieck groups equivariant. The natural integration map by Euler characteristic is replaced by an integration map to the ring of dual numbers:

$$
\mathbb{Q}[\epsilon] /\left(\epsilon^{2}=0\right) .
$$

Date: December 12, 2016. 
Precisely, given a scheme $Z$ with an action by a simple1 abelian variety $A$ we define the integration map by

$$
\mathcal{I}(Z)=e\left(Z^{A}\right)+e\left(\left(Z-Z^{A}\right) / A\right) \epsilon
$$

where $Z^{A}$ is the fix locus of the action, and $e(\cdot)$ is the topological Euler characteristic taken here always in the orbifold sense. This construction arises natural in applications. For example, for a smooth projective variety $X$ of dimension $d$ we have the identity in the Grothendieck ring of varieties

$$
\sum_{n=0}^{\infty}\left[\operatorname{Hilb}^{n}(X)\right] q^{n}=\left(\sum_{n=0}^{\infty}\left[\operatorname{Hilb}^{n}\left(\mathbb{C}^{d}\right)_{0}\right] q^{n}\right)^{[X]}
$$

where $\operatorname{Hilb}^{n}(X)$ is the Hilbert scheme of points on $X$, and Hilb ${ }^{n}\left(\mathbb{C}^{d}\right)_{0}$ is the punctual Hilbert scheme in the affine space $\mathbb{C}^{d}[16]$. In case $X=Y \times A$ where $A$ is a simple abelian variety acting on $X$ by translation in the second factor, a straight-forward argument shows that (11) lifts to the $A$-equivariant Grothendieck ring. Applying our integration map we naturally obtain 2

$$
\begin{aligned}
1+\epsilon \sum_{n=1}^{\infty} e\left(\operatorname{Hilb}^{n}(X) / A\right) q^{n} & =\left(\sum_{n=0}^{\infty} e\left(\operatorname{Hilb}^{n}\left(\mathbb{C}^{d}\right)_{0}\right) q^{n}\right)^{\mathcal{I}(X)} \\
& =\left(\sum_{n=0}^{\infty} P_{d}(n) q^{n}\right)^{\epsilon \cdot e(Y)} \\
& =1+\epsilon \cdot e(Y) \log \left(\sum_{n=0}^{\infty} P_{d}(n) q^{n}\right)
\end{aligned}
$$

where $P_{d}(n)$ is the number of $d$-dimensional partitions of $n$, and we used the convention $f(q)^{\epsilon}=\exp (\log (f) \epsilon)$. The left hand side is (up to a factor) the generating series of Euler characteristics of the generalized Kummer schemes of $Y \times A$, and we recover a formula proven by Shen [30], Morrison-Shen [20], and Gulbrandsen-Ricolfi [14]. In fact, the first order expansion in terms of $e(A)$ was the main motivation that led Gulbrandsen to conjecture (2) for abelian varieties in [13]. Our approach captures this intuition and makes it mathematically rigorous.

1.2. Reduced Donaldson-Thomas invariants. Our main interest here lies in applications to Donaldson-Thomas (DT) invariants 3 for special CalabiYau threefolds $X$. We are particularly interested in the following examples:

(1) $X$ is an abelian threefold, or

\footnotetext{
${ }^{1}$ An abelian variety is simple if all its proper subgroups are 0-dimensional.

${ }^{2}$ See Section 3 for details of the proof.

${ }^{3}$ Donaldson-Thomas invariants are defined by integration over the Hilbert scheme of curves in threefolds and virtually enumerate algebraic curves, see 26] for an introduction.
} 
(2) $X$ is the product of a K3 surface and an elliptic curve $E$.

In both cases an abelian variety acts on the Hilbert schemes by translation and forces almost all ordinary DT invariants to vanish. The definition of DT invariants needs to be modified to be enumerative meaningful.

Let $A$ be an abelian variety which acts on a Calabi-Yau threefold $X$. Let $\operatorname{Hilb}^{n}(X, \beta)$ be the Hilbert scheme of 1-dimensional subschemes $Z \subset X$ satisfying

$$
[Z]=\beta \in H_{2}(X, \mathbb{Z}), \quad \chi\left(\mathcal{O}_{Z}\right)=n \in \mathbb{Z} .
$$

If the induced $A$-action on $\operatorname{Hilb}^{n}(X, \beta)$ has finite stabilizers, we define $A$ reduced Donaldson-Thomas invariants of $X$ by

$$
\mathrm{DT}_{n, \beta}^{X, A \text {-red }}=\int_{\operatorname{Hilb}^{n}(X, \beta) / A} \nu \mathrm{d} e=\sum_{k \in \mathbb{Z}} e\left(\nu^{-1}(k)\right),
$$

where $\nu: \operatorname{Hilb}^{n}(X, \beta) / A \rightarrow \mathbb{Z}$ is Behrend's constructible function [3].

For abelian threefolds (acting on itself by translation) the definition was introduced by Gulbrandsen in [13, where he also showed deformation invariance in many cases. For $\mathrm{K} 3 \times E$ the definition is by Bryan [8] and deformation invariance is proven in [21. In both cases explicit conjectural formulas for the reduced DT invariants are known in all curve classes [22, 10]. The formulas reveal (at least conjecturally and as far as numbers go) rich structures underlying the enumerative geometry of algebraic curves.

In Section 4 we introduce $A$-equivariant versions of Joyce's motivic Hall algebra and equip them with integration maps defined over the ring of dual numbers. This structure is tailored to deal with reduced DT invariants systematically. This leads to new calculations in several interesting cases, and to DT/PT correspondences in previous unknown cases.

1.3. Reduced DT invariants for $K 3 \times E$. Let $S$ be a non-singular projective $\mathrm{K} 3$ surface and let $E$ be a non-singular elliptic curve. We consider the product Calabi-Yau

$$
X=S \times E
$$

on which $E$ acts by translation in the second factor. Using the Künneth decomposition we identify

$$
H_{2}(X, \mathbb{Z})=H_{2}(S, \mathbb{Z}) \oplus H_{2}(E, \mathbb{Z})=H_{2}(S, \mathbb{Z}) \oplus \mathbb{Z} .
$$

The conjectural form of the reduced DT invariants of $X$ is reviewed in Section 2, here we prove the following special case. Define coefficients $\mathrm{m}(d, n)$ by the expansion

$$
\sum_{d=0}^{\infty} \sum_{n \in \mathbb{Z}} \mathrm{m}(d, n) p^{n} t^{d}=-\frac{24 \wp(p, t)}{\prod_{m \geq 1}\left(1-t^{m}\right)^{24}}
$$


where $\wp$ is the Weierstraß elliptic function,

$$
\wp(p, t)=\frac{1}{12}+\frac{p}{(1-p)^{2}}+\sum_{d=1}^{\infty} \sum_{k \mid d} k\left(p^{k}-2+p^{-k}\right) t^{d} .
$$

Theorem 1. For all $d \geq 0$ we have

$$
\exp \left(\sum_{n=1}^{\infty} \mathrm{DT}_{n,(0, d)}^{X, E \text {-red }}(-p)^{n}\right)=\prod_{\ell=1}^{\infty}\left(\frac{1}{1-p^{\ell}}\right)^{\mathrm{m}(d, \ell)} .
$$

Theorem 1 determines all reduced invariants in classes $(0, d) 4$ As in the case of the Hilbert scheme of points we need to exponentiate the generating series of reduced DT invariants to obtain product expansions. The case $d=0$ of Theorem 1 recovers the calculation of reduced degree 0 DT invariants of [30, 20]. For $d>0$ the results give a new and non-trivial check in imprimitive classes for the general multiple cover formula conjectured in [22]. Explicitly, taking the logarithm in the theorem yields the closed formula

$$
\mathrm{DT}_{n,(0, d)}^{X, E-\text { red }}=(-1)^{n} \sum_{k \mid(n, d)} \frac{1}{k} \mathrm{~m}\left(d, \frac{n}{k}\right) .
$$

1.4. Reduced DT invariants for abelian threefolds. Let $A$ be a projective abelian threefold acting on itself by translation. If $n \neq 0$ by deformation invariance the $A$-reduced DT invariants depend only on the typ 5

$$
\left(d_{1}, d_{2}, d_{3}\right), d_{i} \geq 0
$$

of the curve class $\beta$. We write

$$
\mathrm{DT}_{n,\left(d_{1}, d_{2}, d_{3}\right)}^{\mathrm{red}}=\mathrm{DT}_{n, \beta}^{A, A \text {-red }} \text {. }
$$

We restrict here to the degenerate case where $\beta$ has type $(0,0, d)$. If $n<0$ the Hilbert scheme is empty and all reduced invariants vanish. For $n=0$ $A$-reduced invariants are not defined. For $n>0$ we have the following result.

Theorem 2. For all $d \geq 0$ and $n>0$ we have

$$
\mathrm{DT}_{n,(0,0, d)}^{\mathrm{red}}=\frac{(-1)^{n-1}}{n} \sum_{k \mid \operatorname{gcd}(n, d)} k^{2} .
$$

In case $d=0$ the above formula specializes to the degree 0 reduced DT invariants which were conjectured in [13] and proven in [30, 20, 14] using generalized Kummer schemes. If $d>0$ we obtain agreement with the multiple cover formulas of [10, compare Section 2.

\footnotetext{
4 The Hilbert scheme $\operatorname{Hilb}^{n}(X,(0, d))$ is empty for $n<0$ and $E$-invariant for $n=0$.

${ }^{5}$ The type is obtained from the standard divisor theory of the dual abelian variety [10].
} 
1.5. Reduced DT/PT correspondence. A stable pair on a threefold $X$ is the datum $(\mathcal{F}, s)$ of a pure 1-dimensional sheaf $\mathcal{F}$ and a section $s \in H^{0}(\mathcal{F})$ with 0-dimensional cokernel. Following [25] we let $P_{n}(X, \beta)$ be the moduli space of stable pairs with numerical invariants

$$
[\operatorname{Supp}(\mathcal{F})]=\beta \in H_{2}(X, \mathbb{Z}), \quad \chi(\mathcal{F})=n \in \mathbb{Z} .
$$

Let $A$ be an abelian variety which acts on a Calabi-Yau threefold $X$. If the induced action on $P_{n}(X, \beta)$ has finite stabilizers, we define $A$-reduced Pandharipande-Thomas (PT) invariants by

$$
\mathrm{PT}_{n, \beta}^{X, A \text {-red }}=\int_{P_{n}(X, \beta) / A} \nu \mathrm{d} e
$$

where $\nu: P_{n}(X, \beta) / A \rightarrow \mathbb{Z}$ is the Behrend function.

The relationship between usual DT and PT invariants of Calabi-Yau 3folds has been well understood via wall-crossing [32, 6, 35]. For abelian threefolds $A$ we prove in Section 6.4 the following simple correspondence:

$$
\mathrm{DT}_{n, \beta}^{A, A \text {-red }}=\mathrm{PT}_{n, \beta}^{A, A \text {-red }}
$$

for all $n, \beta$ where $A$-reduced invariants are defined.

For $E$-reduced invariants of $\mathrm{K} 3 \times E$ the $\mathrm{DT} / \mathrm{PT}$ correspondence takes a more interesting form. Define generating series of reduced invariants:

$$
\mathrm{DT}_{d}^{\mathrm{red}}(q, t)=\sum_{n, \gamma} \mathrm{DT}_{n,(\gamma, d)}^{X, E-\mathrm{red}} q^{n} t^{\gamma}, \quad \mathrm{PT}_{d}^{\mathrm{red}}(q, t)=\sum_{n, \gamma} \mathrm{PT}_{n,(\gamma, d)}^{X, E-\text {-red }} q^{n} t^{\gamma}
$$

where the sums run over all $n \in \mathbb{Z}$ and all curve classes $\gamma \in H_{2}(S, \mathbb{Z})$ with $(n, \gamma) \neq 0$. Let also

$$
M(q)=\prod_{m=1}^{\infty}\left(1-q^{n}\right)^{-n}
$$

be the MacMahon function, and define coefficients $a_{d}$ by

$$
\sum_{d=0}^{\infty} a_{d} t^{d}=\prod_{m=1}^{\infty}\left(1-t^{m}\right)^{-24} .
$$

By a result of Göttsche [11] we have $a_{d}=e\left(\operatorname{Hilb}^{d}(S)\right)$.

Theorem 3. For all $d \geq 0$,

$$
\exp \left(\mathrm{DT}_{d}^{\mathrm{red}}(q, t)\right)=M(-q)^{-24 a_{d}} \cdot \exp \left(\mathrm{PT}_{d}^{\mathrm{red}}(q, t)\right) .
$$

If $\gamma \neq 0$ then we recover the result of [21],

$$
\mathrm{DT}_{n,(\gamma, d)}^{X, E \text {-red }}=\mathrm{PT}_{n,(\gamma, d)}^{X, E \text {-red }},
$$

while for $\gamma=0$ the correspondence (Theorem 3 ) is new and non-trivial. 
1.6. Relation to other work. (1) The motive of the generalized Kummer schemes were computed in [20] using Grothendieck rings relative to an abelian monoid. It would be interesting to compare this to the motivic class (in the Grothendieck ring of stacks) of the stack quotient $\operatorname{Hilb}^{n}(X) / A$.

(2) The topological vertex method of [9, 8] may yield another approach to Theorems 1 and 2. The method proceeds by stratification and computation of the local invariants. While in principle this method is able to compute the Euler characteristic of the corresponding Hilbert scheme, the difficulty here is to incorporate also the correct Behrend function weights into the computation for DT invariants.

1.7. Plan of the paper. In Section 2 we recall the general multiple cover formulas for abelian threefolds and $\mathrm{K} 3 \times E$ as conjectured in [22, 10]. We also comment on the relationship of Theorem 1 to Gromov-Witten theory. In Section 3 as warmup for the general case we introduce an equivariant Grothendieck ring of varieties and prove the degree 0 cases of Theorems 1 and 2. In Section 4 we introduce the equivariant motivic Hall algebra, which we apply in Section 5 to prove the main theorems following a strategy of Y. Toda [34. In Section 6 we treat the parallel case of abelian threefolds.

1.8. Conventions. We always work over the complex numbers $\mathbb{C}$. All schemes are of finite type, and by definition a variety is a reduced, separated scheme of finite type. A Calabi-Yau threefold is a nonsingular projective threefold $X$ with trivial canonical class $K_{X} \simeq \mathcal{O}_{X}$. In particular the vanishing of $H^{1}\left(X, \mathcal{O}_{X}\right)$ is not required. By the recent work [28, 35] the results of [7, Sec.5] also hold in this more general setting, compare [21, 4.6].

1.9. Acknowledgements. The paper was started when J. S. was visiting MIT in September 2016. We would like to thank Jim Bryan, Andrew Kresch, Davesh Maulik, Rahul Pandharipande, Johannes Schmitt, and Qizheng Yin for their interest and useful discussions.

J. S. was supported by grant ERC-2012-AdG-320368-MCSK in the group of Rahul Pandharipande at ETH Zürich.

\section{Multiple COVER FORMulas}

2.1. Overview. We review here the conjectural formulas for reduced DT invariants of $\mathrm{K} 3 \times E$ by [22] and abelian threefolds by [10].

2.2. $K 3 \times E$. Let $X=S \times E$ be the product of a non-singular projective K3 surface $S$ and an elliptic curve $E$, on which $E$ acts by translation in the second factor. The $E$-reduced DT invariants of $X$ are denoted by

$$
\mathrm{DT}_{n,(\beta, d)}^{\mathrm{red}}=\mathrm{DT}_{n,(\beta, d)}^{X, E \text {-red }}
$$


where $\beta \in H_{2}(S, \mathbb{Z})$ is a (possibly zero) curve class, $d \geq 0$ and $n \in \mathbb{Z}$. Since we require the translation action on the Hilbert scheme to have finite stabilizers we will always require

$$
\beta \neq 0 \text { or } n \neq 0 \text {. }
$$

Define coefficients $c(m)$ by the expansion

$$
\sum_{d \geq 0} \sum_{k \in \mathbb{Z}} c\left(4 d-k^{2}\right) p^{k} t^{d}=24 \phi_{-2,1}(p, t) \wp(p, t)
$$

where $\phi_{-2,1}$ is the unique weak Jacobi form of index 1 and weight -2 ,

$$
\phi_{-2,1}(p, t)=\left(p-2+p^{-1}\right) \prod_{m \geq 1} \frac{\left(1-p t^{m}\right)^{2}\left(1-p^{-1} t^{m}\right)^{2}}{\left(1-t^{m}\right)^{4}}
$$

and $\wp$ is the Weierstraß elliptic function (4). The weight 10 Igusa cusp form is defined by the product expansion

$$
\chi_{10}(p, t, \tilde{t})=p t \tilde{t} \prod_{k, h, d}\left(1-p^{k} t^{h} \tilde{t}^{d}\right)^{c\left(4 h d-k^{2}\right)}
$$

where the product is over all $k \in \mathbb{Z}$ and $h, d \geq 0$ such that

- $h>0$ or $d>0$,

- $h=d=0$ and $k<0$.

We define coefficients $\mathrm{m}(h, d, n)$ by the expansion of the reciprocal of the Igusa cusp form in the region $0<|t|<|p|<1$,

$$
\sum_{h=0}^{\infty} \sum_{d=0}^{\infty} \sum_{n \in \mathbb{Z}} \mathrm{m}(h, d, n) p^{n} t^{h-1} \tilde{t}^{d-1}=\frac{-1}{\chi_{10}(p, t, \tilde{t})} .
$$

The coefficients $\mathrm{m}(h, d, n)$ are related to $\mathrm{m}(d, n)$ introduced before by

$$
\mathrm{m}(d, n)=\mathrm{m}(1, d, n) .
$$

The following conjecture was proposed in [22].

Conjecture 1 ([22]). For all $n, \beta, d$ satisfying $\beta \neq 0$ or $n \neq 0$, we have

$$
(-1)^{n} \mathrm{DT}_{n,(\beta, d)}^{\mathrm{red}}=\sum_{\substack{k \geq 1 \\ k \mid(n, \beta)}} \frac{1}{k} \mathrm{~m}\left(\frac{(\beta / k)^{2}}{2}+1, d, \frac{n}{k}\right)
$$

where $\gamma^{2}=\gamma \cdot \gamma$ is the self-intersection of a class $\gamma \in H_{2}(S, \mathbb{Z})$.

The equality of Conjecture 1 is conjectured to hold for all cases where it is defined. Indeed, the reduced DT invariants on the left hand side are defined if and only if $(\beta, n) \neq(0,0)$ which precisely coincides with the case where the sum on the right hand side makes sense.

If $\beta$ is primitive of square $\beta^{2}=2 h-2$ then (6) says the reduced DT invariant is up to a sign equal to the coefficient $\mathrm{m}(h, d, n)$. If $\beta$ is imprimitive, 
then (6) expresses the reduced DT invariant in terms of primitive invariants. Hence we sometimes refer to (66) as a multiple cover formula. In the most degenerate case $\beta=0$ we recover Theorem 1 .

Finally, for every $d \geq 0$ the rule (6) may be reformulated in the following product expansion:

$$
\exp \left(\sum_{(n, \beta) \neq 0} \mathrm{DT}_{n,(\beta, d)}^{\mathrm{red}}(-p)^{n} t^{\beta}\right)=\prod_{(\ell, \gamma) \neq 0}\left(\frac{1}{1-p^{\ell} t^{\gamma}}\right)^{\mathrm{m}\left(\gamma^{2} / 2+1, d, \ell\right)}
$$

where $(n, \beta)$ and $(\ell, \gamma)$ run over all non-zero pairs of an integer and a (possibly zero) curve class in $H_{2}(S, \mathbb{Z})$.

2.3. Comparision with Gromov-Witten theory. The formula (6) was conjectured in [22] for reduced Gromov-Witten (GW) invariants in curve classes $(\beta, d)$ where $\beta \neq 0$. Translating the statement to DT theory via the conjectural reduced GW/DT correspondence 6 yields Conjecture 1. While reduced GW invariants are not defined for $\beta=0$, the formula makes sense on the DT side and surprisingly gives the correct result.

If $\beta$ vanishes the Donaldson-Thomas generating series is not a rational function and the variable change $p=e^{i u}$ is not well-defined. However, parallel to the case of degree zero DT invariants discussed in [19, 2.1] an asymptotic correspondence may be established as follows.

The analog of the reduced (disconnected) Gromov-Witten potential in case $\beta=0$ and genus $g \geq 2$ is the series

$$
\mathcal{F}^{g}(t)=\int_{S} c_{2}(S) \cdot \prod_{m \geq 1} \frac{1}{\left(1-t^{m}\right)_{S} c_{2}(S)} \sum_{d=0}^{\infty} \frac{1}{2 g-2}\left\langle\tau_{1}(\omega) \lambda_{g-1} \lambda_{g-2}\right\rangle_{g, d}^{E} t^{d}
$$

where $\langle\cdot\rangle_{g, d}^{E}$ are the connected Gromov-Witten invariants of the elliptic curve $E$ in genus $g$ and degree $d$, and $\omega \in H^{2}(E, \mathbb{Z})$ is the class of a point, $\tau_{1}$ is the first descendent insertion, and $\lambda_{k}$ is the $k$-th Chern class of the Hodge bundle. The Euler factor

$$
\prod_{m \geq 1} \frac{1}{\left(1-t^{m}\right)^{\int_{S} c_{2}(S)}}
$$

is the contribution of the non-reduced Gromov-Witten theory of $X$. The factor $2 g-2$ corrects for the integration of the cotangent line bundle over each curve, compare [10, Sec.7]. A calculation by Pixton [27, Prop.4.4.6] based on the results [23, 24] shows

$$
\sum_{d=0}^{\infty}\left\langle\tau_{1}(\omega) \lambda_{g-1} \lambda_{g-2}\right\rangle_{g, d}^{E} t^{d}=(-1)^{g} B_{2 g-2}\left(\begin{array}{c}
2 g \\
2
\end{array}\right) C_{2 g}(t)
$$

\footnotetext{
6 The reduced GW/PT correspondence is conjectured in [22, Conj.D], to which we apply the DT/PT-correspondence of 21].
} 
where $B_{k}$ are the Bernoulli numbers and

$$
C_{k}(t)=-\frac{B_{k}}{k \cdot k !}+\frac{2}{k !} \sum_{n \geq 1} \sum_{\ell \mid n} \ell^{k-1} t^{n}
$$

are renormalized classical Eisenstein series. Let also

$$
\mathcal{F}^{g}(t)=\sum_{d=0}^{\infty} \mathcal{F}_{d}^{g} t^{d}
$$

Then by Theorem 1 the asmptotic Gromov-Witten/Donaldson-Thomas correspondence holds for all $d \geq 0$ :

$$
\sum_{g=2}^{\infty} \mathcal{F}_{d}^{g} u^{2 g-2} \sim c_{d} \cdot \sum_{n=1}^{\infty} \mathrm{DT}_{n,(0, d)}^{\mathrm{red}}(-p)^{n}
$$

under the variable change $p=e^{i u}$, where we have $c_{0}=-1 / 2$, and $c_{d}=-1$ for all $d \geq 1$, and $\sim$ stands for taking the formal expansion on the right hand side, interchanging sums and renormalizing the genus $g \geq 2$ terms via negative zeta values. The overall minus sign in the correspondence (7) corresponds to the difference of the Behrend function of the Hilbert scheme and its quotient by translation. The factor $1 / 2$ in case $d=0$ is parallel (via taking the logarithm) to the square root in the degree 0 asymptotic GW/DT correspondence [19, Eqn.2].

2.4. Abelian 3-folds. Let $A$ be an abelian threefold, and let $\beta \in H_{2}(A, \mathbb{Z})$ be a curve class of type $\left(d_{1}, d_{2}, d_{3}\right)$. Assuming deformation invariance also in the case $n=0$ we will simply write

$$
\mathrm{DT}_{n,\left(d_{1}, d_{2}, d_{3}\right)}^{\mathrm{red}}=\mathrm{DT}_{n,\left(d_{1}, d_{2}, d_{3}\right)}^{A, A-\text { red }}
$$

The translation action on the Hilbert scheme has finite stabilizers (and hence reduced DT invariants are defined) if and only if $n \neq 0$ or at least two of the integers $d_{1}, d_{2}, d_{3}$ are positive.

Define coefficients a $(k)$ by the expansion

$$
\sum_{d=0}^{\infty} \sum_{r \in \mathbb{Z}} \mathrm{a}\left(4 d-r^{2}\right) p^{r} t^{d}=-\phi_{-2,1}(p, t)
$$

where the Jacobi form $\phi_{-2,1}$ was defined in (5). Let also

$$
\mathrm{n}\left(d_{1}, d_{2}, d_{3}, k\right)=\sum_{\delta} \delta^{2}
$$

where $\delta$ runs over all divisors of

$$
\operatorname{gcd}\left(k, d_{1}, d_{2}, d_{3}, \frac{d_{1} d_{2}}{k}, \frac{d_{1} d_{3}}{k}, \frac{d_{2} d_{3}}{k}, \frac{d_{1} d_{2} d_{3}}{k^{2}}\right)
$$

when all numbers in the bracket are integers. 
Conjecture 2 ([10]). If $n>0$ or at least two of the $d_{i}$ are positive, then

$$
(-1)^{n} \mathrm{DT}_{n,\left(d_{1}, d_{2}, d_{3}\right)}^{\mathrm{red}}=\sum_{k} \frac{1}{k} \mathrm{n}\left(d_{1}, d_{2}, d_{3}, k\right) \cdot \mathrm{a}\left(\frac{4 d_{1} d_{2} d_{3}-n^{2}}{k^{2}}\right)
$$

where $k$ runs over all divisors of $\operatorname{gcd}\left(n, d_{1} d_{2}, d_{1} d_{3}, d_{2} d_{3}\right)$ such that $k^{2} \mid d_{1} d_{2} d_{3}$.

For abelian threefolds we obtain product formulas only if $d_{1}=1$ (up to permutation). Assuming Conjecture 2 we have in analogy with the Igusa cusp form

$$
\exp \left(\sum_{d, \tilde{d}=0}^{\infty} \sum_{n \in \mathbb{Z}} \mathrm{DT}_{n,(1, d, \tilde{d})}^{\mathrm{red}}(-p)^{n} t^{d} \tilde{t}^{\tilde{d}}\right)=\prod_{h, d, k}\left(\frac{1}{1-p^{k} t^{h} \tilde{t}^{d}}\right)^{\mathrm{a}\left(4 h d-k^{2}\right)}
$$

where the product is over all $k \in \mathbb{Z}$ and $m_{1}, m_{2} \geq 0$ such that $m_{1}>0$, or $m_{2}>0$, or $m_{1}=m_{2}=0$ and $k>0$.

\section{Equivariant Grothendieck RINGS}

3.1. Overview. As a toy example for the equivariant Hall algebra we introduce the equivariant Grothendieck ring and its integration map to the dual numbers. As application we reprove the following result of [30] and [20].

Let $A$ be an abelian variety and let $Y$ be a non-singular quasi-projective variety. The action of $A$ act on $Y \times A$ by translation in the second factor induces an action on the Hilbert scheme of points $\operatorname{Hilb}^{n}(Y \times A)$ by translation. The quotient

$$
\operatorname{Hilb}^{n}(Y \times A) / A
$$

is a Deligne-Mumford stack for every $n>0$. We also let $d=\operatorname{dim}(Y \times A)$.

Theorem 4. We have

$$
\exp \left(\sum_{n=1}^{\infty} e\left(\operatorname{Hilb}^{n}(Y \times A) / A\right) q^{n}\right)=\left(\sum_{n=0}^{\infty} P_{d}(n) q^{n}\right)^{e(Y)} .
$$

where $P_{d}(n)$ is the number of d-dimensional partitions of $n$.

3.2. Equivariant Grothendieck rings. Let $A$ be a simple abelian variety of dimension $g>0$. The $A$-equivariant Grothendieck group of varieties is the free abelian group $K_{0}^{A}($ Var) generated by the classes

$$
\left[X, a_{X}\right]
$$

of a variety $X$ together with an $A$-action $a_{X}: A \times X \rightarrow X$, modulo the equivariant scissor relations: For every $A$-invariant closed sub-variety $Z \subset X$ with complement $U$,

$$
\left[X, a_{X}\right]=\left[Z,\left.a_{X}\right|_{Z}\right]+\left[U,\left.a_{X}\right|_{U}\right] .
$$


For varieties $X$ and $Y$ with $A$-actions $a_{X}$ and $a_{Y}$ respectively, let $a_{X \times Y}$ be the $A$-action on the product $X \times Y$ obtained from the diagonal $A \rightarrow A \times A$ and the product action $a_{X} \times a_{Y}$. We define a multiplication on $K_{0}^{A}$ (Var) by

$$
\left[X, a_{X}\right] \times\left[Y, a_{Y}\right]=\left[X \times Y, a_{X \times Y}\right] .
$$

The product is commutative and associative with unit

$$
\left[\operatorname{Spec}(\mathbb{C}), a_{\text {triv }}\right]
$$

where $a_{\text {triv }}$ is the trivial $A$-action. We call the pair $\left(K_{0}^{A}(\operatorname{Var}), \times\right)$ the $A$ equivariant Grothendieck ring.

3.3. Schemes. The A-equivariant Grothendieck group of schemes is the free abelian group $K_{0}^{A}(\mathrm{Sch})$ generated by the classes $\left[X, a_{X}\right]$ of a scheme $X$ together with an $A$-action $a_{X}: A \times X \rightarrow X$, modulo the following relations:

(a) $\left[X \sqcup Y, a_{X} \sqcup a_{Y}\right]=\left[X, a_{X}\right]+\left[Y, a_{Y}\right]$ for every pair of schemes $X$ and $Y$ with $A$-actions $a_{X}$ and $a_{Y}$ respectively,

(b) $\left[X, a_{X}\right]=\left[Y, a_{Y}\right]$ for every $A$-equivariant geometric bijection $7 X \stackrel{f}{\rightarrow} Y$. The product on $K_{0}^{A}(\mathrm{Sch})$ is defined identical to the case of varieties. Since the equivariant scissor relation is implied by relations (a) and (b) the natural embedding of the category of varieties into the category of schemes determines a ring homomorphism

$$
K_{0}^{A}(\operatorname{Var}) \rightarrow K_{0}^{A}(\mathrm{Sch}) .
$$

Lemma 1. The morphism (8) is an isomorphism.

Proof. This is parallel to $[7$, Sec.2.3, 2.4]. Let $X$ be a scheme with $A$-action $a_{X}$. We first show the class $\left[X, a_{X}\right]$ is in the image of (8).

By relation (b) we may assume $X$ is reduced. Then there is an affine open $U \subset X$ such that every point of $u$ is seperated in $X 8$ By the valuative criterion, being seperated is invariant under translation by $A$. Hence every point of the translate $U+A$, i.e. the image of $A \times U \stackrel{a_{X}}{\longrightarrow} X$, is seperated, and $U+A$ is a variety. Repeating the argument with the complement of $U+A$, by induction there exists an $A$-equivariant stratification of $X$ by varieties. Thus $X$ admits an $A$-equivariant geometric bijection from a variety $Y$, which by (b) implies the claim.

It remains to check the relations imply each other. The key step is to prove relation (b) follows from the equivariant scissor relation. By stratification we

\footnotetext{
${ }^{7}$ The map $f$ is a geometric bijection if the induced map $f(\mathbb{C}): X(\mathbb{C}) \rightarrow Y(\mathbb{C})$ on $\mathbb{C}$-valued points is a bijection, see [7, Defn.2.7].

${ }^{8}$ Let $\Delta \subset X \times X$ be the diagonal. The non-separated points of $X$ are the closure the image of $\bar{\Delta} \backslash \Delta$ under the projection to the second factor. Hence we may assume $X$ is irreducible. Since $\bar{\Delta} \backslash \Delta$ has dimension strictly less then $X$, the scheme $\bar{\Delta} \backslash \Delta$ does not dominate $X$.
} 
may assume $f: X \rightarrow Y$ is a $A$-equivariant geometric bijection of varieties. Then by the proof of [7, Lem 2.8] there is an open subset $U \subset Y$ such that $f^{-1}(U) \rightarrow U$ is an isomorphism. Since $f$ is $A$-equivariant we may assume $U$ is $A$-invariant. Replacing $X, Y$ by the complement of $U, f^{-1}(U)$ respectively and repeating the argument, the process has to terminate at which point we obtain $\left[X, a_{X}\right]=\left[Y, a_{Y}\right]$ in $K_{0}^{A}(\operatorname{Var})$.

We identify the groups $K_{0}^{A}(\operatorname{Var})$ and $K_{0}^{A}(\mathrm{Sch})$ via the isomorphism (8) .

3.4. Power structures. Recall from [15] that a power structure over a commutative ring $R$ is a map

$$
(1+q R[[q]]) \times R \rightarrow(1+q R[[q]]),
$$

denoted by $(f(q), r) \mapsto f(t)^{r}$, satisfying the following 5 axioms

(1) $f(q)^{0}=1$,

(2) $f(q)^{1}=f(q)$,

(3) $f(q)^{n} \cdot g(q)^{n}=(f(q) \cdot g(q))^{n}$,

(4) $f(q)^{n+m}=f(q)^{n} \cdot f(q)^{m}$,

(5) $f(q)^{n m}=\left(f(q)^{n}\right)^{m}$.

The power structure over the ordinary Grothendieck ring $K_{0}$ (Var) was defined in [15] as follows. Assume $S_{0}$ (Var) is the semi-subring of $K_{0}$ (Var) spanned by effective classes. Let

$$
f(t)=1+\sum_{k \geq 1}\left[M_{k}\right] q^{k}
$$

be a series in $S_{0}(\operatorname{Var})[[q]]$, and $[R]$ be a class in $S_{0}$ (Var). Then $f(t)^{[R]}$ is defined to be the series $1+\sum_{n \geq 1}\left[W_{n}\right] q^{n}$ with

$$
\left.\left[W_{n}\right]=\sum_{\substack{\left(k_{1}, k_{2}, \ldots\right) \\ \sum_{i} i k_{i}=n}}\left[\left(\prod_{i} R^{k_{i}}\right) \backslash \triangle\right) \times \prod_{i} M_{i}^{k_{i}} / \prod_{i} S_{k_{i}}\right],
$$

where $\triangle$ is the big diagonal in $\prod_{i} R^{k_{i}}$, and $S_{k_{i}}$ acts by permuting the corresponding $k_{i}$ factors in $\left(\prod_{i} R^{k_{i}}\right) \backslash \triangle$ and $M_{i}^{k_{i}}$ simultaneously, compare [15]. This defines a power structure over $S_{0}$ (Var) which extends uniquely to a power structure over $K_{0}$ (Var).

We define a power structure on the $A$-equivariant Grothendieck ring $K_{0}^{A}$ (Var) by exactly the same procedure. It only remains, given $A$ actions on $M_{k}$ and $R$ respectively, to endow the classes (9) with $A$-actions. The $A$-actions on $M_{k}$ and $R$ induce a diagonal action on each effective class

$$
\left[\left(\left(\prod_{i} R^{k_{i}}\right) \backslash \triangle\right) \times \prod_{i} M_{i}^{k_{i}} / \prod_{i} S_{k_{i}}\right] .
$$


and we let $\left[W_{k}\right]$ be the associated equivariant effective class in $K_{0}^{A}$ (Var). As in [20, Thm 2.1 and 2.2] this defines a power structure over the semi-ring of $A$-equivariant effective classes, which extends uniquely to $K_{0}^{A}(\mathrm{Var})$.

3.5. Canonical decompositions and $\epsilon$-integration maps I. Let $a_{X}$ be an $A$-action on a variety $X$. Let $U \subset X$ be the closed subset of $A$-fixed points, and let $V=X \backslash U$ be its complement. We call the associated scissor relation

$$
\left[X, a_{X}\right]=\left[U, a_{\text {triv }}\right]+\left[V,\left.a_{X}\right|_{V}\right]
$$

the canonical decomposition of $\left[X, a_{X}\right]$. Since $A$ is a simple abelian group, the induced $A$-action on $V$ has finite stabilizers and the quotient $V / A$ is a Deligne-Mumford stack. We define the $\epsilon$-integration map

$$
\mathcal{I}: K_{0}^{A}(\operatorname{Var}) \rightarrow \mathbb{Q}[\epsilon] / \epsilon^{2}
$$

to be the unique group homomorphism satisfying

$$
\mathcal{I}\left(\left[X, a_{X}\right]\right)=e(U)+e(V / A) \cdot \epsilon
$$

for every variety $X$ with canonical decompostion (10).

Since stratification along stabilizers is compatible with the scissor relation, the canonical decomposition (10) extends uniquely to all classes in $K_{0}^{A}$ (Var), and the map $\mathcal{I}$ is well-defined.

Lemma 2. The $\epsilon$-integration map $\mathcal{I}$ is a ring homomorphism.

Proof. Consider effective classes $\left[X, a_{X}\right]$ and $\left[Y, a_{Y}\right]$ together with their canonical decompositions

$$
\left[X, a_{X}\right]=\left[U_{1}, a_{\text {triv }}\right]+\left[V_{1},\left.a_{X}\right|_{V_{1}}\right]
$$

and

$$
\left[Y, a_{Y}\right]=\left[U_{2}, a_{\text {triv }}\right]+\left[V_{2},\left.a_{Y}\right|_{V_{1}}\right]
$$

The product $\left[X \times Y, a_{X \times Y}\right]$ has the canonical decomposition

$$
\left[X \times Y, a_{X \times Y}\right]=\left[U_{1} \times U_{2}, a_{\text {triv }}\right]+\left[V, a_{V}\right],
$$

with $\left[V, a_{V}\right]=\left[U_{1} \times V_{2}\right]+\left[U_{2} \times V_{1}\right]+\left[V_{1} \times V_{2}\right]$, where we have suppressed the induced $A$-actions. We have

$$
\left(U_{i} \times V_{j}\right) / A \simeq U_{i} \times\left(V_{j} / A\right), \quad\{i, j\}=\{1,2\} .
$$

Since $V_{1} \times V_{2}$ carries an $(A \times A)$-action, the quotient $\left(V_{1} \times V_{2}\right) / A$ carries an $A$-action. Since this action has no fixed points, $e\left(\left(V_{1} \times V_{2}\right) / A\right)=0$. Thus

$$
\begin{aligned}
\mathcal{I}\left(\left[X \times Y, a_{X \times Y}\right]\right) & =e\left(U_{1} \times U_{2}\right)+\epsilon \cdot\left(e\left(U_{1}\right) e\left(V_{2} / A\right)+e\left(U_{2}\right) e\left(V_{1} / A\right)\right) \\
& =\mathcal{I}\left(\left[X, a_{X}\right]\right) \cdot \mathcal{I}\left(\left[Y, a_{Y}\right]\right) .
\end{aligned}
$$


For $f \in 1+q \mathbb{Q}[\epsilon][[q]]$ and $g \in \mathbb{Q}[\epsilon]$ we let $f^{g}=e^{g \cdot \log (f)}$ where the logarithm is defined by the formal expansion $\log (1+x)=-\sum_{n \geq 1}(-x)^{n} / n$. The associated power structure on $\mathbb{Q}[\epsilon]$ is compatible with $\mathcal{I}$ as follows:

Lemma 3. Let $Y$ be a variety, and let a be the A-action on $Y \times A$ by translation in the second factor. Then

$$
\mathcal{I}\left(\left(\frac{1}{1-q}\right)^{[Y \times A, a]}\right)=\left(\frac{1}{1-q}\right)^{e(Y) \cdot \epsilon} .
$$

Proof. We expand the motivic zeta function:

$$
\left(\frac{1}{1-q}\right)^{[Y \times A, a]}=1+\sum_{n \geq 1}\left[(Y \times A)^{(n)}, a^{(n)}\right] \cdot q^{n},
$$

where $(Y \times A)^{(n)}$ is the $n$-th symmetric product of $Y \times A$ and $a^{(n)}$ is the induced $A$-action. Hence it suffices to show

$$
e\left((Y \times A)^{(n)} / A\right)=\frac{e(Y)}{n} .
$$

Let $\pi:(Y \times A)^{(n)} \rightarrow A$ be the composition of the projection $(Y \times A)^{(n)} \rightarrow$ $A^{(n)}$ and the addition map $A^{(n)} \rightarrow A$. By [20, Lem 28 and 29], we have

$$
e\left(\pi^{-1}\left(0_{A}\right)\right)=e(Y) \cdot n^{2 g-1}
$$

where $0_{A} \in A$ is the zero. The stack $(Y \times A)^{(n)} / A$ is the quotient of $\pi^{-1}\left(0_{A}\right)$ by the group $A[n]$ of $n$-torsion points on $A$. Hence

$$
e\left((Y \times A)^{(n)} / A\right)=\frac{e\left(\pi^{-1}\left(0_{A}\right)\right)}{n^{2 g}}=\frac{e(Y)}{n} .
$$

3.6. Proof of Theorem 4, By a topological argument, see [30, Prop 2.1], the Euler characteristic $e\left(\operatorname{Hilb}^{n}(Y \times A) / A\right)$ does not depend on the choice of the abelian variety $A$. Hence we may assume $A$ is simple.

Let $H_{n}=\operatorname{Hilb}^{n}\left(\mathbb{C}^{d}\right)_{0}$ be the punctual Hilbert scheme of length $n$ in $\mathbb{C}^{d}$, and let $\left[H_{n}\right]$ be its class in $K_{0}^{A}$ (Var) (with the trivial $A$-action). Let $a$ be the $A$-action on $Y \times A$ by translation in the second factor, and let $a^{[n]}$ be the induced action on $\operatorname{Hilb}^{n}(Y \times A)$. By the stratification of $\operatorname{Hilb}^{n}(Y \times A)$ (compare [16, 12]) we have

$$
\sum_{n=0}^{\infty}\left[\operatorname{Hilb}^{n}(Y \times A), a^{[n]}\right] q^{n}=\left(\sum_{m=0}^{\infty}\left[H_{m}\right] q^{m}\right)^{[Y \times A, a]} .
$$

We apply the $\epsilon$-integration map to the equation (111). Since there exits classes $\left[M_{i}\right] \in K_{0}^{A}(\operatorname{Var})$ with trivial $A$-actions such that

$$
\sum_{m \geq 0}\left[H_{m}\right] q^{m}=\prod_{m \geq 1}\left(\frac{1}{1-q^{m}}\right)^{\left[M_{m}\right]}
$$


by Lemmas 2 and 3 the integration map of the righthand side of (11) is compatible with the power structure. It follows

$$
1+\sum_{n \geq 1} e\left(X_{n}\right) q^{n} \cdot \epsilon=\left(\sum_{m \geq 0} P_{d}(m) q^{m}\right)^{e(Y) \epsilon} .
$$

Theorem 4 is deduced by comparing the coefficient of $\epsilon$.

3.7. Degree 0 DT invariants. Let $S$ be a non-singular K3 surface, let $E$ be an elliptic curve, and let $A$ be an abelian threefold.

Corollary 1. For all $n>0$,

$$
\mathrm{DT}_{n, 0}^{S \times E, E-\mathrm{red}}=24 \frac{(-1)^{n-1}}{n} \sum_{\ell \mid n} \ell^{2}, \quad \mathrm{DT}_{n, 0}^{A, A-\mathrm{red}}=\frac{(-1)^{n-1}}{n} \sum_{\ell \mid n} \ell^{2},
$$

Proof. This follows by [4, Theorem 4, and MacMahon's formula for 3dimensional partitions,

$$
\sum_{m \geq 0} P_{3}(m) q^{m}=\prod_{m \geq 1}\left(1-t^{m}\right)^{-m} .
$$

\section{Equivariant motivic Hall algebras}

4.1. Overview. Let $A$ be a simple abelian variety of dimension $g>0$. In this section we introduce the $A$-equivariant motivic hall algebra of $X$ and its integration map over the ring of dual numbers. Applying results of Joyce we define reduced generalized Donaldson-Thomas invariants, and prove a structure result for reduced DT invariants generalizing results of Toda and Bridgeland.

4.2. Modified Grothendieck rings. The modified $A$-equivariant Grothendieck group $\widetilde{K}_{0}^{A}($ Var $)$ is the $\mathbb{Q}$-vector space $K_{0}^{A}(\operatorname{Var}) \otimes \mathbb{Q}$ modulo the following extra relations:

(E) Let $X_{1}, X_{2}$, and $Y$ be varieties with $A$-actions $a_{1}, a_{2}$, and $a_{Y}$ respectively. If all $A$-actions have finite stabilizers and $X_{i} \rightarrow Y(i=1,2)$ are $A$-equivariant Zariski fibrations with the same fibers, then

$$
\left[X_{1}, a_{1}\right]=\left[X_{2}, a_{2}\right] \in \widetilde{K}_{0}^{A}(\operatorname{Var}) .
$$

Lemma 4. Under the assumptions of relation (E), we have

$$
e\left(X_{1} / A\right)=e\left(X_{2} / A\right) \text {. }
$$

Proof. Let $W$ be the fiber of both fibrations. The $A$-eqivariant fibration $X_{i} \rightarrow Y$ induces a map $f_{i}: X_{i} / A \rightarrow Y / A$ of Deligne-Mumford stacks with constant fiber $W$. Hence for $i=1,2$ we have

$$
e\left(X_{i} / A\right)=e(W) \cdot e(Y / A) .
$$


The ring structure on $K_{0}^{A}(\operatorname{Var})$ induces naturally a ring structure on $\widetilde{K}_{0}^{A}$ (Var). By Lemma 4 the integration map $\mathcal{I}$ descends to a well-defined ring homomorphism

$$
\mathcal{I}: \widetilde{K}_{0}^{A}(\operatorname{Var}) \rightarrow \mathbb{Q}[\epsilon]
$$

4.3. Preliminaries. We will follow Bridgeland [7] for the discussion of Grothendieck groups of stacks and motivic Hall algebras. In particular, all stacks here are assumed to be algebraic and locally of finite type with affine geometric stabilizers. Geometric bijections and Zariski fibrations of stacks are defined in [7, Def 3.1] and [7, Def 3.3] respectively.

Let $\sigma: G \times \mathcal{X} \rightarrow \mathcal{X}$ be a group action on a stack $\mathcal{X}$, and let $x: \operatorname{Spec} \mathbb{C} \rightarrow \mathcal{X}$ be a $\mathbb{C}$-valued point of $\mathcal{X}$. The inertia subgroup $\operatorname{In}(x)$ of $x$ is defined by the fiber product

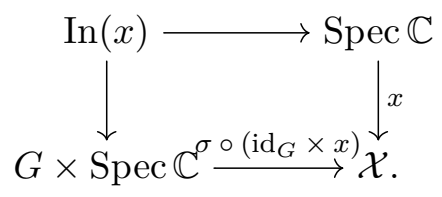

The stabilizer group of the point $x \in \mathcal{X}$ is the fibered product

$$
\operatorname{Iso}(x)=\operatorname{Spec} \mathbb{C} \times_{x, \mathcal{X}, x} \operatorname{Spec} \mathbb{C} .
$$

The stabilizer group of the $G$-action at $x$ is the quotient

$$
S(x)=\operatorname{In}(x) / \operatorname{Iso}(x) .
$$

We refer to [29] for a discussion of group actions on stacks.

4.4. Equivariant Grothendieck group of stacks. The following is the main definition of Section 4, and the equivariant analog of [7, Defn.3.10].

Definition 1. Let $\mathcal{S}$ be an algebraic stack equipped with an $A$-action $a_{\mathcal{S}}$. The relative Grothendieck group of stacks $K_{0}^{A}(\mathrm{St} / \mathcal{S})$ is defined to be the $\mathbb{Q}$-vector space generated by the classes

$$
\left[\mathcal{X} \stackrel{f}{\rightarrow} \mathcal{S}, a_{\mathcal{X}}\right]
$$

where $\mathcal{X}$ is an algebraic stack of finite type, $a_{\mathcal{X}}$ is an $A$-action on $\mathcal{X}$, and $f$ is an $A$-equivariant morphism, modulo the following relations:

(a) For every pair of stacks $\mathcal{X}_{1}$ and $\mathcal{X}_{2}$ with $A$-actions $a_{1}$ and $a_{2}$ respectively a relation

$$
\left[\mathcal{X}_{1} \sqcup \mathcal{X}_{2} \stackrel{f_{1} \sqcup f_{2}}{\longrightarrow} \mathcal{S}, a_{1} \sqcup a_{2}\right]=\left[\mathcal{X}_{1} \stackrel{f_{1}}{\longrightarrow} \mathcal{S}, a_{1}\right]+\left[\mathcal{X}_{2} \stackrel{f_{2}}{\longrightarrow} \mathcal{S}, a_{2}\right]
$$

where $f_{i}(i=1,2)$ are $A$-equivariant. 
(b) For every commutative diagram

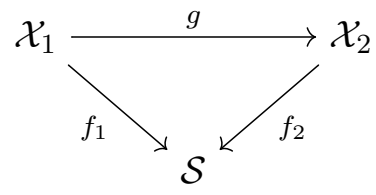

with all morphisms $A$-equivariant and $g$ a geometric bijection a relation

$$
\left[\mathcal{X}_{1} \stackrel{f_{1}}{\longrightarrow} \mathcal{S}, a_{1}\right]=\left[\mathcal{X}_{2} \stackrel{f_{2}}{\longrightarrow} \mathcal{S}, a_{2}\right] .
$$

(c) Let $\mathcal{X}_{1}, \mathcal{X}_{2}, \mathcal{Y}$ be stacks equipped with $A$-actions $a_{1}, a_{2}, a_{Y}$ respectively satisfying one of the following conditions:

(i) the $A$-actions $a_{1}, a_{2}, a_{Y}$ have stabilizers $A$ at every $\mathbb{C}$-point.

(ii) the $A$-actions $a_{1}, a_{2}, a_{Y}$ have finite stabilizers at every $\mathbb{C}$-point. Then for every pair of $A$-equivariant Zariski fibrations

$$
h_{1}: \mathcal{X}_{1} \rightarrow \mathcal{Y}, \quad h_{2}: \mathcal{X}_{2} \rightarrow \mathcal{Y}
$$

with the same fibers and for every $A$-equivariant morphism $\mathcal{Y} \stackrel{g}{\rightarrow} \mathcal{S}$, a relation

$$
\left[\mathcal{X}_{1} \stackrel{g \circ h_{1}}{\longrightarrow} \mathcal{S}, a_{1}\right]=\left[\mathcal{X}_{2} \stackrel{g \circ h_{2}}{\longrightarrow} \mathcal{S}, a_{2}\right]
$$

Remark. In relation (c) the stabilizer group of all actions must have the same type (i.e. either finite or $A$ ) for the integration maps to behave reasonable. For example, we require the classes

$$
\left[A \rightarrow \operatorname{Spec} \mathbb{C}, a_{\text {triv }}\right], \quad\left[A \rightarrow \operatorname{Spec} \mathbb{C}, a_{A}\right]
$$

where $a_{\text {triv }}$ is the trivial action and $a_{A}$ is the action of $A$ on itself by translation, to be different in $K_{0}^{A}(\mathrm{St} / \mathrm{Spec} \mathbb{C})$.

4.5. Absolute Grothendieck group of stacks. We define the absolute $A$-equivariant Grothendieck group of stacks by

$$
K_{0}^{A}(\mathrm{St})=K_{0}^{A}(\mathrm{St} / \mathrm{Spec} \mathbb{C}) .
$$

The product of stacks and the diagonal action makes $K_{0}^{A}(\mathrm{St})$ a commutative ring. Since relation (E) of Section 4.2 is a special case of relation (c) of Definition 1, the inclusion of the category of varieties into the category of stacks naturally yields a map

$$
\widetilde{K}_{0}^{A}(\operatorname{Var}) \rightarrow K_{0}^{A}(\mathrm{St})
$$

For all $d \geq 1$ consider the classes of the general linear group $\mathrm{GL}_{d}$ endowed with the trivial $A$-action,

$$
\left[\mathrm{GL}_{d}\right] \in \widetilde{K}_{0}^{A}(\mathrm{Var})
$$


By relation (c) (compare [7, 3.3]) the image of $\left[\mathrm{GL}_{d}\right]$ is invertible in $K_{0}^{A}(\mathrm{St})$. We then have the following structure result for $K_{0}^{A}(\mathrm{St})$.

Proposition 1. The morphism (12) induces an isomorphism

$$
\widetilde{K}_{0}^{A}(\operatorname{Var})\left[\left[\mathrm{GL}_{d}\right]^{-1}, d \geq 1\right] \stackrel{\simeq}{\longrightarrow} K_{0}^{A}(\mathrm{St}) \text {. }
$$

For the proof we will require the following lemma.

Lemma 5. Let $\mathcal{X}$ be a stack with an A-action such that every $\mathbb{C}$-point of $\mathcal{X}$ has finite stabilizers. Then there exist a variety $Y$ with an $A$-action and a $G=\mathrm{GL}_{d}$ action such that both actions commute, and an A-equivariant geometric bijection

$$
f: Y / G \rightarrow \mathcal{X}
$$

Proof of Lemma 5. Since the $A$-action on $\mathcal{X}$ has finite stabilizers at $\mathbb{C}$-valued points, the quotient stack $\mathcal{X} / A$ also has affine stabilizers. By [7, Prop 3.5] applied to $\mathcal{X} / A$ we obtain a geometric bijection

$$
g: Y / G \rightarrow \mathcal{X} / A .
$$

with $Y$ a variety and $G=\mathrm{GL}_{d}$ for some $d$. Form the Cartesian diagrams

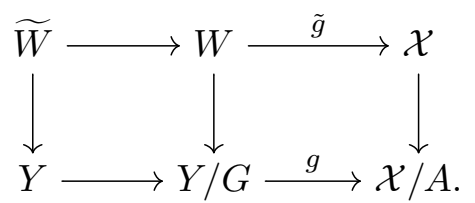

Since $g$ is a geometric bijection also $\tilde{g}$ is a geometric bijection. Since $\widetilde{W} \rightarrow Y$ is a $G$-equivariant $A$-torsor, and $\widetilde{W} \rightarrow W$ is an $A$-equivariant $G$-torsor, the induced actions of $A$ and $G$ on $\widetilde{W}$ commute. This also shows $W=\widetilde{W} / G$.

Since $\widetilde{W} \rightarrow Y$ is an $A$-torsor over the variety $Y$, we have $\widetilde{W}$ is an algebraic space, and we obtain the $A$-equivariant geometric bijection

$$
\widetilde{W} / G \rightarrow \mathcal{X}
$$

Finally we need to replace the algebraic space $\widetilde{W}$ by a variety $V$. This can be achieved by using a similar stratification argument as in the proof of Lemma 1. Since every algebraic space has an open subspace represented by an affine scheme, we may choose a subvariety $U \subset \widetilde{W}$ such that the total $(A \times G)$-orbit of $U$ is represented by a variety. Taking the complement and repeating, we can stratify $\widetilde{W}$ by $(A \times G)$-equivariant varieties $U_{i} \subset \widetilde{W}$. Hence we set $V=\sqcup U_{i}$ and obtain a geometric bijection $V / G \rightarrow \mathcal{X}$.

Proof of Proposition [1. We construct an inverse $R$ to (13). Let $\mathcal{X}$ be a stack with $A$-action $a_{\mathcal{X}}$. Consider the stratification

$$
\mathcal{X}=\mathcal{U} \sqcup \mathcal{V}
$$


such that the stabilizer of every $\mathbb{C}$-point of $\mathcal{U}$ (resp. of $\mathcal{V}$ ) is $A$ (a finite group). By relations (b) and (a) we find

$$
\left[\mathcal{X}, a_{\mathcal{X}}\right]=\left[\mathcal{U},\left.a_{\mathcal{X}}\right|_{\mathcal{U}}\right]+\left[\mathcal{V}, a_{\mathcal{X}} \mid \mathcal{V}\right]
$$

By relation $(\mathrm{c}$, ii) with $\mathcal{Y}=\operatorname{Spec} \mathbb{C}$ we have

$$
\left[\mathcal{U}, a_{\mathcal{X}} \mid \mathcal{U}\right]=\left[\mathcal{U}, a_{\text {triv }}\right]
$$

where $a_{\text {triv }}$ is the trivial action. Hence we may assume either the $A$-action on $\mathcal{X}$ is trivial, or has finite stabilizers. In the first case, let $Y / \mathrm{GL}_{d} \rightarrow \mathcal{X}$ be a geometric bijection with $Y$ a variety [7, Prop 3.5]; then set

$$
R\left(\left[\mathcal{X}, a_{\text {triv }}\right]\right)=\left[Y, a_{\text {triv }}\right] /\left[\mathrm{GL}_{d}\right] .
$$

If the $A$-action on $\mathcal{X}$ has finite stabilizers, let $Y / \mathrm{GL}_{d} \rightarrow \mathcal{X}$ be the $A$ equivariant geometric bijection of Lemma 5; then we set

$$
R\left(\left[\mathcal{X}, a_{\text {triv }}\right]\right)=\left[Y, a_{Y}\right] /\left[\mathrm{GL}_{d}\right] .
$$

It remains to check $R$ is well-defined and preserves the relations (a,b,c). This follows along the lines of [7, Lem.3.9] from Lemma 5, and matching the relation (c) with the extra relation (E) imposed on $\widetilde{K}_{0}^{A}(\operatorname{Var})$.

4.6. Hall algebras. Let $A$ be a non-trivial simple abelian variety, let $X$ be a non-singular projective Calabi-Yau threefold and let

$$
a_{X}: A \times X \rightarrow X
$$

be a free action. Let $\operatorname{Coh}(X)$ be the category of coherent sheaves on $X$, and let $\mathcal{M}$ be the moduli stack of objects in $\operatorname{Coh}(X)$. The abelian variety $A$ acts on $\operatorname{Coh}(X)$ by translation by $a_{X}$, which induces an $A$-action

$$
a_{\mathcal{M}}: A \times \mathcal{M} \rightarrow \mathcal{M}
$$

The equivariant motivic Hall algebra $\left(H^{A}(X), *\right)$ of $X$ is defined to be the relative Grothendieck group

$$
H^{A}(X):=K_{0}^{A}(\mathrm{St} / \mathcal{M}) .
$$

with the product $*$ defined by extensions of coherent sheaves as follows. Let $\mathcal{M}^{(2)}$ be the moduli stack of short exact sequences

$$
E_{\bullet}: 0 \rightarrow E_{1} \rightarrow E_{2} \rightarrow E_{3} \rightarrow 0 .
$$

The stack $\mathcal{M}^{(2)}$ carries an $A$-action $a_{\mathcal{M}^{(2)}}$ induced by $a_{X}$, and $A$-equivariant projections $p_{i}: \mathcal{M}^{(2)} \rightarrow \mathcal{M}$ defined by $p_{i}\left(E_{\bullet}\right)=E_{i}$ for $i=1,2,3$. Given $A$-equivariant morphisms

$$
\left[\mathcal{X} \stackrel{g_{1}}{\rightarrow} \mathcal{M}, a_{\mathcal{X}}\right] \text { and }\left[\mathcal{Y} \stackrel{g_{2}}{\rightarrow} \mathcal{M}, a_{\mathcal{Y}}\right] .
$$


consider the Cartesian diagram

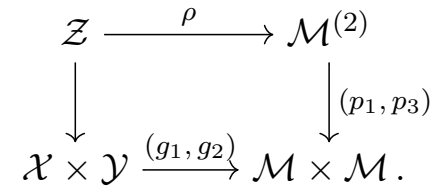

The morphism $\rho$ is $A$-equivariant with respect to the natural diagonal $A$ action $a_{\mathcal{Z}}$ on $\mathcal{Z}$. We define the Hall algebra product $*$ by

$$
\left[\mathcal{X} \stackrel{g_{1}}{\rightarrow} \mathcal{M}, a_{\mathcal{X}}\right] *\left[\mathcal{Y} \stackrel{g_{2}}{\longrightarrow} \mathcal{M}, a_{\mathcal{Y}}\right]=\left[\mathcal{Z} \stackrel{p_{2} \circ \rho}{\longrightarrow} \mathcal{M}, a_{\mathcal{Z}}\right] .
$$

The unit of $\left(H^{A}(X), *\right)$ is the point $[\operatorname{Spec}(\mathbb{C}) \rightarrow \mathcal{M}]$ corresponding to the trivial sheaf $0 \in \operatorname{Coh}(X)$ (together with the trivial $A$-action).

The Hall algebra $H^{A}(X)$ is naturally a $K_{0}^{A}(\mathrm{St})$-module via

$$
\left[\mathcal{Y}, a_{\mathcal{Y}}\right] \cdot\left[\mathcal{Z} \rightarrow \mathcal{M}, a_{\mathcal{Z}}\right]:=\left[\mathcal{Y} \times \mathcal{Z} \rightarrow \mathcal{M}, a_{\mathcal{Y} \times \mathcal{Z}}\right]
$$

where the $A$-action $a_{\mathcal{Y} \times \mathcal{Z}}$ is induced by the diagonal $A \rightarrow A \times A$ and the product action $a_{\mathcal{Y}} \times a_{\mathcal{Z}}$.

4.7. Regular classes and Poisson algebras. Let $\mathbb{L} \in \widetilde{K}_{0}^{A}(\operatorname{Var})$ be the class of the affine line (with the trivial $A$-action), which we view also as an element in $K_{0}^{A}(\mathrm{St})$ via the morphism (12). Consider the ring

$$
\Lambda=\widetilde{K}_{0}^{A}(\operatorname{Var})\left[\mathbb{L}^{-1},\left(\mathbb{L}^{n}+\ldots+1\right)^{-1}, n \geq 1\right]
$$

We define $H_{\mathrm{reg}}^{A}(X)$ to be the $\Lambda$-submodule of $H^{A}(X)$ generated by the classes $\left[Z \rightarrow \mathcal{M}, a_{Z}\right]$ where $Z$ is a variety with an $A$-action $a_{Z}$. The elements in $H_{\text {reg }}^{A}(X)$ are called regular.

Proposition 2. The $\Lambda$-submodule of regular elements $H_{\mathrm{reg}}^{A}(X)$ is closed under the Hall algebra product *,

$$
H_{\mathrm{reg}}^{A}(X) * H_{\mathrm{reg}}^{A}(X) \subset H_{\mathrm{reg}}^{A}(X),
$$

and hence a $\Lambda$-algebra. Moreover, the quotient

$$
H_{\mathrm{sc}}^{A}(X):=H_{\mathrm{reg}}^{A}(X) /(\mathbb{L}-1) H_{\mathrm{reg}}^{A}(X)
$$

is a commutative $\widetilde{K}_{0}^{A}(\operatorname{Var})$-algebra.

We will prove Proposition 2 in Section 4.9.

The algebra $H_{\mathrm{sc}}^{A}(X)$ is called the equivariant semi-classical Hall algebra. Identical to the non-equivariant case, Proposition 2 implies that the Poisson bracket on $H_{\text {reg }}^{A}(X)$ defined by

$$
\{f, g\}:=\frac{f * g-g * f}{\mathbb{L}-1}, \quad f, g \in H_{\mathrm{reg}}^{A}(X)
$$

induces a Poisson bracket on the equivariant semi-classical Hall algebra $H_{\mathrm{sc}}^{A}(X)$. Hence $\left(H_{\mathrm{sc}}^{A}(X), *,\{\},\right)$ is a Poisson algebra. 
4.8. Canonical decompositions and $\epsilon$-integration maps II. We define an integration map on the Poisson algebra $\left(H_{\mathrm{sc}}^{A}(X), *,\{\},\right)$.

Let $K(X)$ be the Grothendieck group of coherent sheaves on $X$, and let $\Gamma$ be the image of the Chern character map

$$
\Gamma=\operatorname{Im}\left(\operatorname{ch}: K(X) \rightarrow H^{*}(X, \mathbb{Q})\right) .
$$

The Euler pairing $\chi($,$) on \operatorname{Coh}(X)$ descends to the Euler form

$$
\chi: \Gamma \times \Gamma \rightarrow \Gamma \text {. }
$$

Consider the abelian group

$$
C^{\epsilon}(X):=\bigoplus_{v \in \Gamma} \mathbb{Q}[\epsilon] \cdot c_{v}
$$

where $\epsilon^{2}=0$. The product

$$
c_{v_{1}} * c_{v_{2}}=(-1)^{\chi\left(v_{1}, v_{2}\right)} c_{v_{1}+v_{2}}
$$

and the Poisson bracket

$$
\left\{c_{v_{1}}, c_{v_{2}}\right\}=(-1)^{\chi\left(v_{1}, v_{2}\right)} \chi\left(v_{1}, v_{2}\right) c_{v_{1}+v_{2}}
$$

make $\left(C^{\epsilon}(X), *,\{\},\right)$ a Poisson algebra.

The stack $\mathcal{M}$ splits as a disjoint union of open and closed substacks

$$
\mathcal{M}=\bigsqcup_{v \in \Gamma} \mathcal{M}_{v}
$$

according to Chern characters in $\Gamma$. Hence the equivariant Hall algebra admits the $\Gamma$-graded decomposition

$$
H^{A}(\mathcal{M})=\bigoplus_{v \in \Gamma} H_{v}^{A}(\mathcal{M})
$$

where $H_{v}^{A}(\mathcal{M})$ is spanned by $A$-equivariant classes factoring through $\mathcal{M}_{v}$.

Parallel to (10) for any $A$-equivariant effective regular class

$$
\left[Z \rightarrow \mathcal{M}, a_{Z}\right] \in H_{\mathrm{reg}}^{A}(X)
$$

with $Z$ a variety, we define the canonical decomposition to be

$$
\left[Z \rightarrow \mathcal{M}, a_{Z}\right]=\left[U \rightarrow \mathcal{M}, a_{\text {triv }}\right]+\left[V \rightarrow \mathcal{M},\left.a_{Z}\right|_{V}\right]
$$

where $U$ is the closed subset formed by $A$-fixed points and $V=Z \backslash U$. Since $\left.a_{Z}\right|_{V}$ has finite stablizers, the quotient $V / A$ is a Deligne-Mumford stack. We define the $\epsilon$-integration map

$$
\mathcal{I}: H_{\mathrm{sc}}^{A}(X) \rightarrow C^{\epsilon}(X) .
$$

to be the unique group homomorphism such that for every effective class $\left[Z \stackrel{g}{\rightarrow} \mathcal{M}, a_{Z}\right] \in H_{v}^{A}(\mathcal{M})$ with canonical decomposition (16) we have

$$
\mathcal{I}\left(\left[Z \stackrel{g}{\rightarrow} \mathcal{M}, a_{Z}\right]\right)=\left(\int_{U} g^{*} \nu_{\mathcal{M}} \mathrm{d} e+(-1)^{\operatorname{dim} A}\left(\int_{V / A} g^{*} \nu_{\mathcal{M}} \mathrm{d} e\right) \cdot \epsilon\right) \cdot c_{v}
$$


where $\nu_{\mathcal{M}}$ is the Behrend function on $\mathcal{M}$ and the second integral is defined by

$$
\int_{V / A} g^{*} \nu_{\mathcal{M}} \mathrm{d} e=\sum_{k \in \mathbb{Z}} k \cdot e\left(\left(g^{*} \nu_{\mathcal{M}}\right)^{-1}(k) / A\right) .
$$

Since the Behrend function is constant along $A$-orbits, (18) is well-defined.

To show $\mathcal{I}$ is well-defined we need to check the morphism is compatible with the relations (a-c) of Section 4.4 restricted to regular classes. Since we can stratify the stack $\mathcal{M}$ by values of the Behrend function, we only need to consider regular classes $\alpha \in K_{0}^{A}(\mathrm{St} / \mathcal{M})$ over a sub-stack

$$
\mathcal{M}_{\tau} \subset \mathcal{M}
$$

where the Behrend function is constant. Then by projecting $\alpha$ to an element in $K_{0}^{A}(\mathrm{St})$ and using Proposition 1 and [7, Lem.3.8] we obtain that $\mathcal{I}$ is welldefined, compare [7, 7.2].

Theorem 5. $\mathcal{I}: H_{\mathrm{sc}}^{A}(X) \rightarrow C^{\epsilon}(X)$ is a Poisson algebra homomorphism.

4.9. Proof of Proposition 2 and Theorem 5. Both proofs rely on a stratification developed in [7, Prop 6.2] whose $A$-equivariant form is the following.

Proposition 3. Let $Y_{1}$ and $Y_{2}$ be varieties with A-actions. Assume we have A-equivariant morphisms

$$
f_{1}: Y_{1} \rightarrow \mathcal{M}, \quad f_{2}: Y_{2} \rightarrow \mathcal{M},
$$

and let

$$
\mathcal{E}_{i} \in \operatorname{Coh}\left(Y_{i} \times X\right)(i=1,2)
$$

be the corresponding families of sheaves on $X$. Then we can stratify $Y_{1} \times Y_{2}$ by locally clased $A$-invariant sub-varieties $W \subset Y_{1} \times Y_{2}$, such that for each closed point $w \in W$ the vector spaces

$$
\operatorname{Ext}_{X}^{k}\left(\left.\mathcal{E}_{2}\right|_{w \times X},\left.\mathcal{E}_{1}\right|_{w \times X}\right)
$$

have fixed dimensions $d_{k}(W)$, and if we form the Cartesian diagrams

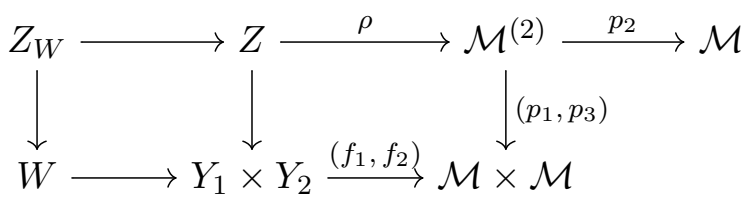

then there exist an A-equivariant Zariski $\mathbb{C}^{d_{1}(W)}$-bundle $Q \rightarrow W$ such that

$$
Z_{W} \simeq\left[Q / \mathbb{C}^{d_{0}(W)}\right]
$$

where $\mathbb{C}^{d_{0}(W)}$ acts trivially. 
Proof. In [7, Prop 6.2], the subsets $W$ are chosen to be affine. But since extension groups form locally trivial bundles along $A$-orbits, we may instead also use the $A$-orbits $\cup_{a \in A}(W+a)$ in the proof of [7, Prop 6.2].

Proof of Proposition 2. The proof is parallel to that of [7, Thm 5.1], but we spell it out here to present the general method. Let

$$
y_{i}:=\left[Y_{i} \stackrel{f_{i}}{\longrightarrow} \mathcal{M}, a_{Y_{i}}\right], \quad i=1,2
$$

be equivariant regular classes defined by $f_{i}$ as in Proposition 3 , Consider the $A$-equivariant stratification as in Proposition 3 ,

$$
Y_{1} \times Y_{2}=\bigsqcup_{j} W_{j}
$$

By definition and the diagram (19) we have

$$
y_{1} * y_{2}=\sum_{j}\left[Z_{W_{j}} \rightarrow \mathcal{M}\right]
$$

where the morphisms is the first row of (19). Hence (20) yields

$$
y_{1} * y_{2}=\sum_{j} \mathbb{L}^{-d_{0}\left(W_{j}\right)}\left[Q_{j} \stackrel{g_{i}}{\rightarrow} \mathcal{M}\right]
$$

where $g_{i}$ is the bundle induced by the universal extension, and we have supressed all $A$-actions for clarity. Since the right-hand side of (21) is regular, we have proved the first part of Proposition 2.

We prove the second part. Since the complement of the zero-section of $Q_{j} \rightarrow W_{j}$ is a Zariski $\mathbb{C}^{*}$-fibration over $\mathbb{P}\left(Q_{j}\right)$ by relation (c) of Definition 1 we have

$$
\left[Q_{j} \stackrel{g_{j}}{\rightarrow} \mathcal{M}\right]=\left[W_{j} \rightarrow \mathcal{M}\right]+[\mathbb{L}-1]\left[\mathbb{P}\left(Q_{j}\right) \rightarrow \mathcal{M}\right]
$$

with compatible $A$-actions. Hence by (21) we have

$$
y_{1} * y_{2}=\sum_{j}\left[W_{j} \rightarrow \mathcal{M}\right]=\left[Y_{1} \times Y_{2} \stackrel{\phi}{\rightarrow} \mathcal{M}\right] \quad \bmod (\mathbb{L}-1),
$$

where $\phi$ is induced by $Y_{1} \times Y_{2} \stackrel{\left(f_{1}, f_{2}\right)}{\longrightarrow} \mathcal{M} \times \mathcal{M}$ and

$$
\mathcal{M} \times \mathcal{M} \rightarrow \mathcal{M},\left(\left[\mathcal{E}_{1}\right],\left[\mathcal{E}_{2}\right]\right) \mapsto\left[\mathcal{E}_{1} \oplus \mathcal{E}_{2}\right] .
$$

Since (23) is independent of the order of multiplication, $*$ is commutative.

Proof of Theorem 5. For equivariant effective classes

$$
y_{i}:=\left[Y_{i} \stackrel{f_{i}}{\rightarrow} \mathcal{M}, a_{Y_{i}}\right], i=1,2
$$

we need to check the product identity

$$
\mathcal{I}\left(y_{1} * y_{2}\right)=\mathcal{I}\left(y_{1}\right) * \mathcal{I}\left(y_{2}\right)
$$


and the Poisson bracket identity

$$
\mathcal{I}\left(\left\{y_{1}, y_{2}\right\}\right)=\left\{\mathcal{I}\left(y_{1}\right), \mathcal{I}\left(y_{2}\right)\right\}
$$

By stratification of $\mathcal{M}$ we may assume $f_{i}$ maps into the substack $\mathcal{M}_{n_{i}} \subset \mathcal{M}$ of objects with a fixed Chern character $v_{i}$ such that the Behrend function on $\mathcal{M}_{n_{i}}$ is constant with value $n_{i}$. We may further assume that the effective classes $y_{i}$ are of one of the following types:

Type 1. Every $\mathbb{C}$-valued point on $Y_{i}$ is $A$-fixed with respect to $a_{Y_{i}}$.

Type 2. Every $\mathbb{C}$-valued point on $Y_{i}$ has finite stabilizers with respect to $a_{Y_{i}}$. We follow the calculations of [7, Section 7.2] to treat each case.

Case 1. Both $y_{1}$ and $y_{2}$ are of Type 1 . Then the $A$-actions does not play a role and the $\epsilon$-term does not appear. The proof of [7, Thm 5.2] applies.

Case 2. Assume $y_{1}$ is of Type 1 and $y_{2}$ is of Type 2. Then by definition

$$
\mathcal{I}\left(y_{1}\right)=n_{1} e\left(Y_{1}\right) \cdot c_{v_{1}}, \quad \mathcal{I}\left(y_{2}\right)=(-1)^{\operatorname{dim} A} n_{2} e\left(Y_{2} / A\right) \epsilon \cdot c_{v_{2}},
$$

where the quotient $Y_{2} / A$ is induced by the action $a_{Y_{2}}$. By (23) and the first Behrend function identity in [35, Thm 2.6] we have

$$
\mathcal{I}\left(y_{1} * y_{2}\right)=(-1)^{\operatorname{dim} A}(-1)^{\chi\left(v_{1}, v_{2}\right)} n_{1} n_{2} \cdot e\left(Y_{1} \times Y_{2} / A\right) \epsilon \cdot c_{v_{1}+v_{2}} .
$$

We obtain the identity (24) by (14) and

$$
e\left(Y_{1}\right) e\left(Y_{2} / A\right)=e\left(Y_{1} \times Y_{2} / A\right) .
$$

The calculation of $\mathcal{I}\left(\left\{y_{1}, y_{2}\right\}\right)$ is similar. Let $\widehat{Q}_{j} \stackrel{\hat{g}}{\rightarrow} W_{j}$ be the Zariski bundle induced by the extension

$$
\operatorname{Ext}^{1}\left(\mathcal{E}_{1}, \mathcal{E}_{2}\right), \quad\left(\left[\mathcal{E}_{1}\right],\left[\mathcal{E}_{2}\right]\right) \in \operatorname{Im}\left(f_{1}, f_{2}\right) \subset \mathcal{M} .
$$

By the expression (21), the relation (22), and Serre duality, we get

$$
\begin{aligned}
\left\{y_{1}, y_{2}\right\}=\sum_{j}\left(\left(d_{3}\left(W_{j}\right)-d_{0}\left(W_{j}\right)\right)\right. & \cdot\left[W_{j} \rightarrow \mathcal{M}\right] \\
+ & {\left.\left[\mathbb{P}\left(Q_{j}\right) \rightarrow \mathcal{M}\right]-\left[\mathbb{P}\left(\widehat{Q}_{j}\right) \rightarrow \mathcal{M}\right]\right) }
\end{aligned}
$$

where we have supressed the natural $A$-actions on the right hand side. The second Behrend function identity in [35, Thm 2.6] yields

$$
\begin{aligned}
\mathcal{I}\left(\left\{y_{1}, y_{2}\right\}\right) & =(-1)^{\operatorname{dim} A}\left(\sum_{j}(-1)^{\chi\left(v_{1}, v_{2}\right)} \chi\left(v_{1}, v_{2}\right) n_{1} n_{2} \cdot e\left(W_{j} / A\right) \epsilon\right) c_{v_{1}+v_{2}} \\
& =(-1)^{\operatorname{dim} A}(-1)^{\chi\left(v_{1}, v_{2}\right)} n_{1} n_{2} \chi\left(v_{1}, v_{2}\right) \cdot e\left(Y_{1} \times Y_{2} / A\right) \epsilon \cdot c_{v_{1}+v_{2}}
\end{aligned}
$$

which coincides with the right-hand side of (25) by (15).

Case 3. Both $y_{1}$ and $y_{2}$ are of Type 2. Since $\epsilon^{2}=0$ we have

$$
\mathcal{I}\left(y_{1}\right) * \mathcal{I}\left(y_{2}\right)=\left\{\mathcal{I}\left(y_{1}\right), \mathcal{I}\left(y_{2}\right)\right\}=0 .
$$


On the other hand, both equations (26) and (27) also hold in this case. The product $Y_{1} \times Y_{2}$ carries an $(A \times A)$-action with no fixed points, hence $e\left(Y_{1} \times Y_{2} / A\right)=0$, and we have

$$
\mathcal{I}\left(y_{1} * y_{2}\right)=\mathcal{I}\left(\left\{y_{1}, y_{2}\right\}\right)=0 .
$$

4.10. Generalized DT invariants. Let $\mathcal{L}$ be a fixed polarization on $X$. The slope function

$$
\mu_{\mathcal{L}}(\mathcal{E})=\frac{\operatorname{ch}_{3}(\mathcal{E})}{c_{1}(\mathcal{L}) \cdot \operatorname{ch}_{2}(\mathcal{E})}
$$

defines a stability condition on the category $\mathrm{Coh}_{\leq 1}(X)$ of sheaves with support of dimension $\leq 1$. Let

$$
v_{n, \beta}=(0,0, \beta, n) \in \Gamma \subset \bigoplus_{i=0}^{3} H^{2 i}(X, \mathbb{Z}),
$$

be a non-zero numerical class and consider the moduli stack

$$
\mathcal{M}_{n, \beta} \subset \mathcal{M}
$$

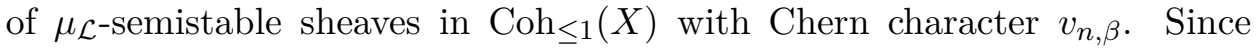
semi-stability is preserved by translation the $A$-action on $\mathcal{M}$ restricts to an action $a_{\mathcal{M}_{n, \beta}}$ on $\mathcal{M}_{n, \beta}$. We define

$$
\delta_{n, \beta}^{A}=\left[\mathcal{M}_{n, \beta} \hookrightarrow \mathcal{M}, a_{\mathcal{M}_{n, \beta}}\right] \in H^{A}(X)
$$

and take the formal logarithm

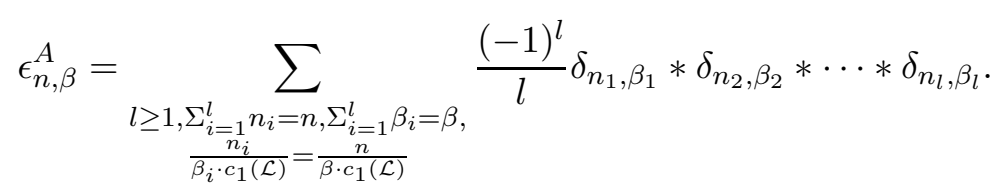

The following theorem is the equivariant analog of Joyce's no pole theorem [18, Thm.8.7], see also [5] for a modern proof.

Theorem 6. The element $(\mathbb{L}-1) \epsilon_{n, \beta}^{A} \in H^{A}(X)$ is regular, i.e,

$$
(\mathbb{L}-1) \epsilon_{n, \beta}^{A} \in H_{\text {reg }}^{A}(X) .
$$

Proof. We prove the Theorem by making Joyce's virtual projection operators [17. $A$-equivariant. For this we work with the $A$-equivariant Hall algebra which satisfies relations (a) and (b) of Section 4.4, but not (c). The key step here is that every stack

$$
\mathcal{M}_{n, \beta} \hookrightarrow \mathcal{M}
$$

admits a $A$-equivariant geometric bijection

$$
f: Y / G \rightarrow \mathcal{M}_{n, \beta},
$$


where $Y$ is a variety with an $A$-action and a $G$-action which commute. Since the virtual projection operators are explicitly defined on $Y / G$ and $A$ equivariant, the projection on virtual indecomposable objects is well-defined on $\mathcal{M}_{n, \beta}$ and yields an $A$-equivariant and virtual indecomposable object. Its image in $H_{\text {reg }}^{A}(X)$ is precisely (29) and hence $(\mathbb{L}-1) \epsilon_{n, \beta}^{A}$ is regular.

To show (30) we can stratify $\mathcal{M}_{n, \beta}$ into a component $\mathcal{U}$ where the action has finite stabilizers, and a component $\mathcal{V}$ where the action has stabilizer group $A$ at every closed point. The claim follows for the first component by Lemma 5, and we only need to consider the second. If $A$ has dimension $\geq 2$ then there does not exist a 1-dimensional sheaf fixed by $A$ and $\mathcal{V}$ is empty. Hence we may assume $A$ is an elliptic curve. Since the $A$ action on $X$ is free, the stack quotient

$$
S=X / A
$$

is a non-singular proper algebraic space of dimension 2 and hence a nonsingular projective surface. Let $\pi: X \rightarrow S$ be the quotient map and let $F$ be the class of a fiber of $\pi$. Then $\mathcal{V}$ is empty unless $\beta=d F$ and $n=0$ for some $d>0$, in which case let $\mathcal{N}_{d}$ be the moduli stack of 0 -dimensional sheaves of length $d$ on $S$, equipped with the trivial $A$-action. Then pullback via $\pi$ induces an $A$-equivariant geometric bijection onto $\mathcal{V}$,

$$
\mathcal{N}_{d} \rightarrow \mathcal{V} \subset \mathcal{M}_{0, d F}
$$

The claim then follows from Kresch's stratification result [7, Prop 3.5] applied to $\mathcal{N}_{d}$, and equipping $Y$ with the trivial $A$-action.

Let $(\mathbb{L}-1) \epsilon_{n, \beta}^{A}$ denote also the projection of

$$
(\mathbb{L}-1) \epsilon_{n, \beta}^{A} \in H_{\mathrm{reg}}^{A}(X)
$$

on the equivariant semi-classical Hall algebra $H_{\mathrm{sc}}^{A}(X)$.

Proposition 4. There exists $N_{n, \beta}^{\mathrm{red}} \in \mathbb{Q}$ such that

$$
\mathcal{I}\left((\mathbb{L}-1) \epsilon_{n, \beta}^{A}\right)=-\left(N_{n, \beta}^{\mathrm{red}} \cdot \epsilon\right) \cdot c_{v_{n, \beta}} .
$$

Proof. By the definition of $\mathcal{I}$ we have

$$
\mathcal{I}\left((\mathbb{L}-1) \epsilon_{n, \beta}^{A}\right)=-\left(N_{n, \beta}+N_{n, \beta}^{\mathrm{red}} \cdot \epsilon\right) \cdot c_{v_{n, \beta}} .
$$

where $N_{n, \beta} \in \mathbb{Q}$ is the generalized DT invariant of [6, 31, 35] and $N_{n, \beta}^{\mathrm{red}} \in \mathbb{Q}$.

If $\operatorname{dim}(A)>1$ then no non-trivial sheaf of dimension $\leq 1$ is $A$-invariant. Hence $N_{n, \beta}=0$ for all $(n, \beta) \neq 0$.

If $\operatorname{dim}(A)=1$ then every $A$-invariant sheaf is supported on the elliptic curve $A$ which implies $N_{n, \beta}=0$ by [33, Lem. 2.11] 9

\footnotetext{
${ }^{9}$ We may also use [34, Prop 6.7] here.
} 
4.11. Wall-crossing formulas. We define a reduced version of the invariants $L_{n, \beta}$ defined in [6, 31, 35]. We follow the discussion in [35, 4.2].

Let $D^{b} \operatorname{Coh}(X)$ be the bounded derived category of coherent sheaves on $X$, and consider the slope function

$$
\nu_{\mathcal{L}}(\mathcal{E}):=\frac{c_{1}(\mathcal{E}) \cdot \mathcal{L}^{2}}{\operatorname{rk}(\mathcal{E})}, \quad \mathcal{E} \in \operatorname{Coh}(X)
$$

which defines a weak stability condition on $\operatorname{Coh}(X)$. Let $\mathcal{A}$ be the category of complexes $I^{\bullet} \in D^{b} \operatorname{Coh}(X)$ satisfying the following conditions:

(a) $h^{i}\left(I^{\bullet}\right)=0$ if $i \neq 0,1$.

(b) All Harder-Narasimhan factors of $h^{0}\left(I^{\bullet}\right)$ have slopes $\leq 0$.

(c) All Harder-Narasimhan factors of $h^{1}\left(I^{\bullet}\right)$ have slopes $>0$.

By [35, 3.3] the category $\mathcal{A}$ is the tilt of $\operatorname{Coh}(X)$ along a torsion pair and hence abelian. Let $\mathcal{M}_{\mathcal{A}}$ be the moduli stack of objects in the category $\mathcal{A}$, and let

$$
a_{\mathcal{M}_{\mathcal{A}}}: A \times \mathcal{M}_{\mathcal{A}} \rightarrow \mathcal{M}_{\mathcal{A}}
$$

be the natural translation action by $A$. Then we can define the $A$-equivariant motivic Hall algebra $H^{A}(\mathcal{A})$ for $\mathcal{M}_{\mathcal{A}}$ parallel to $\mathcal{M}$. By [35, Thm 1.1] the $\epsilon$-integration map

$$
\mathcal{I}: H_{\mathrm{sc}}^{A}(\mathcal{A}) \rightarrow C^{\epsilon}(X)
$$

defined as in (17) is a homomorphism of Poisson algebras. We refer to [35, Sec.2] for a detailed discussion about how to replace $\mathcal{M}$ by $\mathcal{M}_{\mathcal{A}}$.

Recall the slope function (28) and let $\mathcal{M}_{n, \beta}^{L} \subset \mathcal{M}_{\mathcal{A}}$ be the moduli stack of objects $I^{\bullet} \in \mathcal{A}$ satisfying the following conditions:

(a) $\operatorname{ch}\left(I^{\bullet}\right)=u_{n, \beta}:=(1,0,-\beta,-n)$.

(b) $h^{0}\left(I^{\bullet}\right)$ is an ideal sheaf.

(c) $h^{1}\left(I^{\bullet}\right) \in \operatorname{Coh}_{\leq 1}(X)$ and $\mu_{\mathcal{L}}(\mathcal{E}) \geq 0$ for every sub-sheaf $\mathcal{E} \subset h^{1}\left(I^{\bullet}\right)$.

(d) $\operatorname{Hom}\left(\mathcal{E}[-1], I^{\bullet}\right)=0$ for any $\mathcal{E} \in \operatorname{Coh}(X)$ with $\mu_{\mathcal{L}}(\mathcal{E}) \geq 0$.

Let

$$
\mathcal{L}_{n, \beta}^{A}=\left[\mathcal{M}_{n, \beta}^{L} \subset \mathcal{M}_{\mathcal{A}},\left.a_{\mathcal{M}_{\mathcal{A}}}\right|_{\mathcal{M}_{n, \beta}^{L}}\right] \in H^{A}\left(\mathcal{M}_{\mathcal{A}}\right)
$$

be the class defined by the moduli stack $\mathcal{M}_{n, \beta}^{L}$, with the $A$-action obtained by the restriction of $a_{\mathcal{M}_{\mathcal{A}}}$. Then by [35, 4.2] the class

$$
(\mathbb{L}-1) \mathcal{L}_{n, \beta}^{A} \in H^{E}\left(\mathcal{M}_{\mathcal{A}}\right)
$$

is a regular element. Define invariants $L_{n, \beta}, L_{n, \beta}^{\mathrm{red}} \in \mathbb{Q}$ by

$$
\mathcal{I}\left((\mathbb{L}-1) \mathcal{L}_{n, \beta}^{A}\right)=-\left(L_{n, \beta}+L_{n, \beta}^{\mathrm{red}} \cdot \epsilon\right) c_{u_{n, \beta}} .
$$

By construction $L_{n, \beta} \in \mathbb{Z}$ coincide with the usual L-invariant defined in [35].

Lemma 6. For every $\beta$ we have 
(1) $L_{n, \beta}^{\mathrm{red}}=L_{-n, \beta}^{\mathrm{red}}$ for all $n \in \mathbb{Z}$,

(2) $L_{n, \beta}^{\text {red }}=0$ if $n \gg 0$.

Proof. By the same proof as in [35, Sec.4] since the dualizing functor is $A$-equivariant.

In (3) we defined $A$-reduced Donaldson-Thomas invariants if the action of $A$ on the Hilbert scheme has finite stabilizers. Here we extend the definition to the general case as follows. For all $n, \beta$, let

$$
V_{n, \beta} \subset \operatorname{Hilb}^{n}(X, \beta)
$$

be the complement of the fixed locus of translation by $A$. Then we define

$$
\mathrm{DT}_{n, \beta}^{X, A \text {-red }}=\int_{V_{n, \beta} / A} \nu \mathrm{d} e
$$

where $\nu: V_{n, \beta} / A \rightarrow \mathbb{Z}$ is the Behrend function on the quotient.

The following Theorem is the analog for reduced invariants of the main structure result of Donaldson-Thomas theory [6, 31, 35].

Theorem 7. We have the following formula,

$$
\begin{aligned}
& \sum_{n, \beta} \mathrm{DT}_{n, \beta}^{X, A-\mathrm{red}} q^{n} t^{\beta} \\
& \quad=\left(\sum_{n>0, \beta}(-1)^{n-1} n N_{n, \beta}^{\mathrm{red}} q^{n} t^{\beta}\right) \cdot\left(\sum_{n, \beta} L_{n, \beta} q^{n} t^{\beta}\right)+\sum_{n, \beta} L_{n, \beta}^{\mathrm{red}} q^{n} t^{\beta} .
\end{aligned}
$$

Proof. By a straightforward argument the identity [35, Thm 4.8] lifts to the $A$-equivariant motivic hall algebra. Applying the reduced integration map $\mathcal{I}$ by Proposition 4 the left-hand side of (32) is the $\epsilon$-coefficient of

$$
\exp \left(\sum_{n>0, \beta}(-1)^{n-1} n N_{n, \beta}^{\mathrm{red}} \epsilon q^{n} t^{\beta}\right) \cdot\left(\sum_{n, \beta}\left(L_{n, \beta}+L_{n, \beta}^{\mathrm{red}} \epsilon\right) q^{n} t^{\beta}\right) .
$$

\section{REDUCED DT invariants OF $K 3 \times E$}

5.1. Overview. Let $X$ be the product of a K3 surface $S$ and an elliptic curve $E$. We let $E$ act on $X$ by translation in the second factor. Throughout the section all reduced invariants shall be understood as E-reduced invariants. In particular we write

$$
\mathrm{DT}_{n, \beta}^{\mathrm{red}}=\mathrm{DT}_{n, \beta}^{X, E-\mathrm{red}}
$$




\section{2. $L$-invariants.}

Proposition 5. We have $L_{n,(0, d)}^{\mathrm{red}}=0$ for all $n$ and $d \geq 0$, and

$$
\sum_{d \geq 0} \sum_{n \in \mathbb{Z}} L_{n,(0, d)} q^{n} t^{d}=\prod_{m \geq 1}\left(1-t^{m}\right)^{-24}
$$

Proof. Equality (34) is proven in [34, Prop 6.8], hence it remains to show all $L_{n,(0, d)}^{\text {red }}$ vanish. If $n<0$ the Hilbert scheme $\operatorname{Hilb}^{n}(X,(0, d))$ is empty. If $n=0$ we have

$$
\operatorname{Hilb}^{0}(X,(0, d)) \simeq \operatorname{Hilb}^{d}(S) .
$$

which is invariant under the $E$-action, see the proof of [34, Prop 6.8]. Hence $\mathrm{DT}_{n,(0, d)}^{\text {red }}=0$ for $n \leq 0$. By Theorem 7 we conclude $L_{n,(0, d)}^{\text {red }}=0$ for all $n \leq 0$, from which the result follows by Lemma 6 .

5.3. Reduced N-invariants. The proof of the following result is a modification of an argument by Toda, see [34, Prop 6.7].

Proposition 6. Let $n>0, d \geq 0$ and $k=\operatorname{gcd}(n, d)$. Then

$$
N_{n,(0, d)}^{\mathrm{red}}=\left(\frac{k}{n}\right)^{2} N_{k, 0}^{\mathrm{red}}=24 \cdot \frac{1}{n^{2}} \sum_{\ell \mid k} \ell^{2} .
$$

Proof. By (32) we have

$$
\mathrm{DT}_{n, 0}^{\mathrm{red}}=(-1)^{n-1} n N_{n, 0}^{\mathrm{red}} .
$$

Hence the case $d=0$ follows from Corollary 1 .

Assume $d>0$, and let $\mathcal{M}_{n,(0, d)}$ be the moduli space of $\mathcal{L}$-semistable sheaves of Chern character $(0,0,(0, d), n)$, see Section 4.10 .

If $k=\operatorname{gcd}(n, d)=1$ then every semistable sheaf $\mathcal{E}$ is stable and hence

$$
\mathcal{E}=j_{*} \mathcal{E}^{\prime}
$$

for a stable sheaf $\mathcal{E}^{\prime}$ supported on $j: E_{s} \hookrightarrow X$ for some $s \in S$, where $E_{s}=s \times E$. By the classification [1, 2] we conclude

$$
\mathcal{M}_{n,(0, d)} \cong \mathcal{M}_{1,(0,0)}
$$

where, under the identification of $E$ with its dual $\operatorname{Pic}^{0}(E)$, the isomorphism is given by taking the determinant on each fiber $E_{s}$,

$$
\mathcal{E}=j_{*} \mathcal{E}^{\prime} \mapsto j_{*} \operatorname{det}\left(\mathcal{E}^{\prime}\right) .
$$

It remains to compare the translation action by $E$ on both sides of (35). Let $t_{a}: X \rightarrow X,(s, e) \mapsto(s, e+a)$ be the translation by an element $a \in E$. We have

$$
\operatorname{det}\left(t_{a}^{*} \mathcal{E}\right)=\operatorname{det}(\mathcal{E}) \otimes \mathcal{O}_{E_{s}}(-n a)=t_{n a}^{*} \operatorname{det}(\mathcal{E})
$$


Hence the isomorphism (35) is $E$-equivariant with respect to $n$-times the natural translation action on the right hand side. Taking into account the stabilizers group $E[n]$ of $n$-torsion points of $E$, we conclude

$$
N_{n,(0, d)}^{\mathrm{red}}=\frac{1}{n^{2}} \cdot N_{1,(0,0)}^{\mathrm{red}}=\frac{24}{n^{2}}
$$

which verifies the proposition in case $k=1$.

Assume $m=k m_{0}$ and $d=k d_{0}$ with $\operatorname{gcd}\left(m_{0}, d_{0}\right)=1$. Then according to [1, 2] there is no stable object in $\mathcal{M}_{n,(0, d)}$. Every semistable sheaf $\mathcal{E}$ in $\mathcal{M}_{n,(0, d)}$ has exactly $k$ Jordan-Hölder(JH) factors, and each factor determines a $\mathbb{C}$-valued point in $\mathcal{M}_{n_{0},\left(0, d_{0}\right)}$. The universal family on $M_{n_{0},\left(0, d_{0}\right)} \times_{S} X$ induces a derived equivalence

$$
D^{b} \operatorname{Coh}(X) \stackrel{\simeq}{\rightarrow} D^{b} \operatorname{Coh}(X)
$$

sending $[\mathcal{E}] \in \mathcal{M}_{n_{0},\left(0, d_{0}\right)}$ to a skyscraper sheaf $\mathbb{C}_{p}$ for some $p \in X$. Hence comparing Jordan-Hölder factors we obtain the isomorphism

$$
\mathcal{M}_{n,(0, d)} \stackrel{\simeq}{\rightarrow} \mathcal{M}_{k,(0,0)} \text {. }
$$

Applying the same argument as in the case $k=1$ to each JH-factor, the isomorphism (37) is $E$-equivariant with respect to the $n_{0}$ times the natural translation on $\mathcal{M}_{k,(0,0)}$. Hence

$$
N_{n,(0, d)}^{\mathrm{red}}=\frac{1}{n_{0}^{2}} N_{k,(0,0)}^{\mathrm{red}}
$$

and the claim follows from case $d=0$.

5.4. Proof of Theorem 1, By Theorem 7 the reduced DT invariants are completely determined by the $L$, the reduced $L$, and the reduced $N$ invariants. Hence Theorem 1 follows from Propositions 6 and 5 .

5.5. Proof of Theorem 3. The Hall algebra identity of [35, Lem 3.16] lifts to the $A$-equivariant Hall algebra. Applying the $\epsilon$-integration map shows that the difference between the generating series of reduced DT and PT invariants is

$$
\left(\sum_{n>0}(-1)^{n-1} n N_{n, 0}^{\mathrm{red}} q^{n}\right) \cdot\left(\sum_{n, \beta} L_{n, \beta} q^{n} t^{\beta}\right) .
$$

By definition, the ordinary L-invariant $L_{n, \beta}$ vanishes if $\beta$ is not of the form $(0, d)$ since there is no $E$-fixed point in the moduli space $\mathcal{M}_{n, \beta}^{L}$. Hence if $\gamma \in H_{2}(S, \mathbb{Z})$ is non-zero then

$$
\mathrm{DT}_{n,(\gamma, d)}^{\mathrm{red}}=\mathrm{PT}_{n,(\gamma, d)}^{\mathrm{red}} .
$$


Finally, by Propositions 6 and 5 we obtain

$$
\mathrm{DT}_{n,(0, d)}^{\mathrm{red}}=\mathrm{PT}_{n,(0, d)}^{\mathrm{red}}+24\left[\log \left(\prod_{n \geq 1}\left(1-(-q)^{n}\right)^{n}\right) \prod_{m \geq 1}\left(1-t^{m}\right)^{-24}\right]_{q^{n} t^{d}} \text {. }
$$

\section{REDUCED DT INVARIANTS FOR ABELIAN 3-FOLDS}

6.1. Overview. Let $B$ be an non-singular simple principally polarized abelian surface, let $E$ be an elliptic curve and let

$$
A=B \times E .
$$

Here we compute the $A$-reduced DT invariant of $X$ in class

$$
(0, d) \in H_{2}(A, \mathbb{Z}) .
$$

By deformation invariance [13] this yields Theorem 2,

Since $A$ is not simple the equivariant Hall algebra methods of Section 4 can not be applied directly and need to be modified. In particular we need to account for more complicated stabilizer groups. For $A=B \times E$ this leads to an integration map which takes values in the ring

$$
\mathbb{Q}\left[\epsilon_{1}, \epsilon_{2}\right] /\left(\epsilon_{1}^{2}=\epsilon_{2}^{2}=0\right) .
$$

6.2. Equivariant Hall algebra. The following Lemma asserts that all stabilizer groups of $A$ can be controlled.

Lemma 7. Every subgroup $G$ of $A$ is of the form (a) $G=A$, (b) $G=B \times K$, (c) $G=K^{\prime} \times E$, or (d) $G=K^{\prime \prime}$ for finite groups $K, K^{\prime}, K^{\prime \prime}$.

Proof. Every subgroup $G \subset A$ has finitely many connected components all of which have the same dimension. Let $G^{\circ}$ be the connected component of $G$ containing the zero.

If $G^{\circ}$ has dimension 0, the group $G$ is of type (d).

If $G^{\circ}$ is of dimension 1, then $B$ simple implies that the projection $G^{\circ} \rightarrow B$ is constant. Hence $G$ is of type (c).

If $G^{\circ}$ is of dimension 2, consider the projection $\pi: G^{\circ} \rightarrow E$. If $\pi$ is non-constant it is surjective and the kernel is a 1-dimensional subgroup of $B$; a contradiction. Hence $\pi$ is constant and $G$ is of the form (b).

Finally, if $G^{\circ}$ is of dimension 3, the group $G$ is of type (a).

We define the relative and absolute $A$-equivariant Grothendieck group of stacks parallel to Section 4. The Definition 1 is identical except for relation (c). The possible cases (i) and (ii) of stabilizers groups have to replaced with the cases (a), (b), (c), (d) of Lemma 7). This yields an A-equivariant motivic Hall algebra $H^{A}(X)$ resp. $H^{A}(\mathcal{A})$ with the usual properties and structures. 
We define the reduced integration map $\mathcal{I}$. For an effective regular class $\left[Y \rightarrow \mathcal{M}, a_{Y}\right]$ with $Y$ a variety, let

$$
\left[Y \rightarrow \mathcal{M}, a_{Y}\right]=\left[Y^{A}\right]+\left[U_{1}\right]+\left[U_{2}\right]+[V]
$$

be the canonical decomposition, such that every $\mathbb{C}$-point of $Y^{A}, U_{1}, U_{2}, V$ has a stabilizers of type (a), (c), (b), (d) respectively, and we have omitted the natural $A$-actions and the morphism to $\mathcal{M}$ in the notation. Parallel to (17) we define the $\epsilon$-integration map to be the unique group homomorphism

$$
\mathcal{I}: H_{\mathrm{sc}}^{A}(X) \rightarrow \bigoplus_{v \in \Gamma} \mathbb{Q}\left[\epsilon_{1}, \epsilon_{2}\right] /\left(\epsilon_{1}^{2}, \epsilon_{2}^{2}\right) \cdot c_{v}
$$

such that for every regular effective class $\left[Y \rightarrow \mathcal{M}_{v}, a_{Y}\right]$

$$
\begin{aligned}
\mathcal{I}\left(\left[Y \stackrel{g}{\rightarrow} \mathcal{M}, a_{Y}\right]\right) & =\left(\int_{Y^{A}} g^{*} \nu_{\mathcal{M}} \mathrm{d} e+\left(\int_{U_{1} / B} g^{*} \nu_{\mathcal{M}} \mathrm{d} e\right) \epsilon_{1}\right. \\
& \left.-\left(\int_{U_{2} / E} g^{*} \nu_{\mathcal{M}} \mathrm{d} e\right) \epsilon_{2}-\left(\int_{V / A} g^{*} \nu_{\mathcal{M}} \mathrm{d} e\right) \epsilon_{1} \epsilon_{2}\right) \cdot c_{v} .
\end{aligned}
$$

By the same argument as for Theorem [5, the $\epsilon$-integration map (39) is a homomorphism of Poisson algebras.

6.3. Proof of Theorem 2, Let $\epsilon_{n, \beta}^{A}$ be the class in the $A$-equivariant motivic Hall algebra defined in Section 4.10. Define generalized DonaldsonThomas invariants by the reduced integration map of Section 6.2 ,

$$
\mathcal{I}\left((\mathbb{L}-1) \epsilon_{n, \beta}^{A}\right)=-\mathrm{N}_{n, \beta}^{\bullet} \cdot c_{v_{n, \beta}} .
$$

Parallel to Proposition 4 only $A$-reduced invariants are non-zero. We write

$$
\mathbf{N}_{n,(0, d)}^{\bullet}=N_{n,(0, d)}^{A-\text { red }} \epsilon_{1} \epsilon_{2} .
$$

Define equivariant $L$-invariants by $\mathcal{I}\left((\mathbb{L}-1) L_{n, \beta}^{A}\right)=-\mathrm{L}_{n, \beta}^{\bullet} c_{n, \beta}$ and let

$$
\mathrm{DT}_{n, \beta}^{\bullet}=\mathrm{DT}_{n, \beta}+\mathrm{DT}_{n, \beta}^{B \text {-red }} \epsilon_{1}+\mathrm{DT}_{n, \beta}^{E-\text { red }} \epsilon_{2}+\mathrm{DT}_{n, \beta}^{A-\text { red }} \epsilon_{1} \epsilon_{2} .
$$

As in (33) an application of the reduced integration map yields the wallcrossing formula

$$
\sum_{n, \beta} \mathrm{DT}_{n, \beta}^{\bullet} q^{n} t^{\beta}=\exp \left(\sum_{n>0, \beta}(-1)^{n-1} n \mathrm{~N}_{n, \beta}^{\bullet} q^{n} t^{\beta}\right) \cdot\left(\sum_{n, \beta} \mathrm{L}_{n, \beta} q^{n} t^{\beta}\right) .
$$

We have

$$
\begin{cases}\mathrm{DT}_{n, \beta}=0 & \text { if }(n, \beta) \neq 0 \\ \mathrm{DT}_{n, \beta}^{E-\text {-red }}=0 & \text { for all } n, \beta \\ \mathrm{DT}_{n, \beta}^{B-\text { red }}=0 & \text { unless } \beta=(0, d), d>0, n=0 \\ \mathrm{DT}_{0,(0, d)}^{B \text {-red }}=e\left(\operatorname{Hilb}^{d}(B) / B\right) & \text { if } d>0,\end{cases}
$$


which yields

$$
\sum_{n \in \mathbb{Z}} L_{n,(0, d)}^{\bullet} q^{n}= \begin{cases}e\left(\operatorname{Hilb}^{d}(B) / B\right) \epsilon_{1} & \text { if } d>0 \\ 1 & \text { if } d=0 .\end{cases}
$$

Picking out the $q^{n} t^{(0, d)}$ coefficient in (40) hence yields

$$
\mathrm{DT}_{n,(0, d)}^{A \text {-red }}=(-1)^{n-1} n N_{n,(0, d)}^{A-\text { red }} \text {. }
$$

Proposition 7. Let $n>0, d \geq 0$ and $k=\operatorname{gcd}(n, d)$. Then

$$
N_{n,(0, d)}^{A \text {-red }}=\left(\frac{k}{n}\right)^{2} N_{k, 0}^{A \text {-red }}=\frac{1}{n^{2}} \sum_{\ell \mid k} \ell^{2} .
$$

Proof. Since the isomorphisms (35) and (37)) are compatible with $B$-translations the proof of Proposition 6 also shows

$$
N_{n,(0, d)}^{A-\text { red }}=\left(\frac{k}{n}\right)^{2} N_{k, 0}^{A-\text { red }}
$$

Hence the claim follows from the $d=0$ case of (41) and Corollary 1.

Proof of Theorem [. By (41) and Proposition 7.

6.4. DT/PT correspondence. Finally we prove the DT/PT correspondence for abelian 3 -folds. If at least two of the $d_{i}$ are positive in the curve class $\beta=\left(d_{1}, d_{2}, d_{3}\right)$ the $A$-translation on the Chow variety $\operatorname{Chow}(A, \beta)$ has no fixed point. By a comparision of local contribution as in [21] it follows

$$
\mathrm{DT}_{n, \beta}^{A-\text { red }}=\mathrm{PT}_{n, \beta}^{A \text {-red }} \text {. }
$$

The following theorem extends this statement to all classes.

Theorem 8. When $d>0$ and $n>0$, we have

$$
\mathrm{DT}_{n,(0,0, d)}^{A \text {-red }}=\mathrm{PT}_{n,(0,0, d)}^{A \text {-red }} \text {. }
$$

Proof. By deformation invariance we may work with the product $A=B \times E$. Applying the $\epsilon$-integration map of Section 6.2 to the $A$-equivariant version of the Hall algebra identity of [35, Lem 3.16] yields

$$
\sum_{n, d} \mathrm{DT}_{n,(0, d)}^{\bullet} q^{n} t^{d}=\exp \left(\sum_{n>0}(-1)^{n-1} n \mathrm{~N}_{n, 0}^{\bullet} q^{n} t^{d}\right) \cdot\left(\sum_{n, d} \mathrm{PT}_{n,(0, d)}^{\bullet} q^{n} t^{d}\right) .
$$

where the invariants $\mathrm{DT}_{n,(0, d)}^{\bullet}$ and $\mathrm{N}_{n, 0}^{\bullet}$ are defined in Section 6.3 and

$$
\mathrm{PT}_{n,(0, d)}^{\bullet}=\mathrm{PT}_{n,(0, d)}+\mathrm{PT}_{n,(0, d)}^{B \text {-red }} \epsilon_{1}+\mathrm{PT}_{n,(0, d)}^{E \text {-red }} \epsilon_{2}+\mathrm{PT}_{n,(0, d)}^{A \text {-red }} \epsilon_{1} \epsilon_{2} .
$$

By expansion using $\epsilon_{1}^{2}=\epsilon_{2}^{2}=0$ and

$$
\mathrm{PT}_{n,(0, d)}=0
$$

for any $(n, d) \neq 0$, the theorem is deduced. 


\section{REFERENCES}

[1] M. Atiyah, Vector bundles over an elliptic curve, Proc. London Math. Soc. (3) 71957 414-452. 5.3 5.3

[2] L. Bodnarchuk, I. Burban, Y. Drozd, and G. Greuel. Vector bundles and torsion free sheaves on degenerations of elliptic curves, preprint. arXiv:0603261. 5.3, 5.3

[3] K. Behrend, Donaldson-Thomas type invariants via microlocal geometry, Ann. of Math. (2) 170 (2009), no. 3, 1307-1338. 1.2

[4] K. Behrend, B. Fantechi, Symmetric obstruction theories and Hilbert schemes of points on threefolds, Algebra Number Theory 2 (2008), no. 3, 313-345. 3.7

[5] K. Behrend, R. Pooya, The inertia operator on the motivic Hall algebra, arXiv:1612.00372, 4.10

[6] T. Bridgeland, Hall algebras and curve-counting invariants, J. Amer. Math. Soc. 24 (2011), no. 4, 969-998. 1.5, 4.10, 4.114.11

[7] T. Bridgeland, An introduction to motivic Hall algebras, Adv. Math. 229 (2012), no. 1, 102-138. 1.8, 3.3, 7, 4.3, 4.4, 4.5, 4.5, 4.8, 4.9, 4.9, 4.9, 4.10,

[8] J. Bryan, The Donaldson-Thomas theory of $K 3 \times E$ via the topological vertex, arXiv:1504.02920, 1.2, 1.6

[9] J. Bryan and M. Kool, Donaldson-Thomas invariants of local elliptic surfaces via the topological vertex, arXiv:1608.07369, 1.6

[10] J. Bryan, G. Oberdieck, R. Pandharipande, and Q. Yin, Curve counting on abelian surfaces and threefolds, arXiv:1506.00841. 1.2, 1.4, 5, 1.7, 2.1, 2.3, 2

[11] L. Göttsche, The Betti numbers of the Hilbert scheme of points on a smooth projective surface, Math. Ann. 286 (1990), no. 1-3, 193-207. 1.5

[12] L. Göttsche, On the motive of the Hilbert scheme of points on a surface, Math. Res. Lett. 8 (2001), no. 5-6, 613-627. 3.6

[13] M. G. Gulbrandsen, Donaldson-Thomas invariants for complexes on abelian threefolds, Math. Z. 273 (2013), no. 1-2, 219-236. 1.1, 1.2, 1.4, 6.1

[14] M. G. Gulbrandsen and A. Ricolfi, The Euler charateristic of the generalized Kummer scheme of an Abelian threefold, Geom. Dedicata 182 (2016), 73-79. 1.1. 1.4

[15] S. M. Gusein-Zade, I. Luengo, A. Melle-Hernández, A power structure over the Grothendieck ring of varieties, Math. Res. Lett. 11 (2004), no. 1, 49-57. 3.4. 3.4. 3.4

[16] S. M. Gusein-Zade, I. Luengo, A. Melle-HernÃąndez, A power structure over the Grothendieck ring of varieties and generating series of Hilbert schemes of points, Michigan Math. J. 54 (2006), no. 2, 353-359. 1.1. 3.6

[17] D. Joyce, Motivic invariants of Artin stacks and 'stack functions', Q. J. Math. 58 (2007), no. 3, 345-392. 4.10

[18] D. Joyce, Configurations in abelian categories. III. Stability conditions and identities, Adv. Math. 215 (2007), no. 1, 153-219. 4.10

[19] D. Maulik, N. Nekrasov, A. Okounkov, and R. Pandharipande, Gromov-Witten theory and Donaldson-Thomas theory I, Compositio Math. 142 (2006), 1263-1285. 2.3 2.3

[20] A. Morrison and J. Shen, Motivic classes of generalized Kummer schemes via relative power structures, arXiv:1505.02989, 1.1, 1.3, 1.4, 1.6, 3.1, 3.4, 3.5

[21] G. Oberdieck, On reduced stable pair invariants, arXiv:1605.04631, 1.2, 1.5, 1.8, 6, 6.4

[22] G. Oberdieck and R. Pandharipande, Curve counting on $K 3 \times E$, the Igusa cusp form $\chi_{10}$, and descendent integration, in K3 surfaces and their moduli, C. Faber, G. Farkas, and G. van der Geer, eds., Birkhauser Prog. in Math. 315 (2016), 245-278. 1.2. 1.3. 1.7 2.1, 2.2, $1,2.3,6$

[23] A. Okounkov and R. Pandharipande, Gromov-Witten theory, Hurwitz theory, and completed cycles, Ann. of Math. (2) 163 (2006), no. 2, 517-560. 2.3 
[24] A. Okounkov and R. Pandharipande, Virasoro constraints for target curves, Invent. Math. 163 (2006), no. 1, 47-108. 2.3

[25] R. Pandharipande and R. P. Thomas, Curve counting via stable pairs in the derived category, Invent. Math. 178 (2009), no. 2, 407-447. 1.5

[26] R. Pandharipande and R. P. Thomas, 13/2 ways of counting curves, in Moduli spaces, 282-333, London Math. Soc. Lecture Note Ser., 411, Cambridge Univ. Press, Cambridge, 2014. 3

[27] A. Pixton, The Gromov-Witten theory of an elliptic curve and quasimodular forms, Senior Thesis, 2008. 2.3

[28] T. Pantev, B. Töen, M. Vaquíe, and G. Vezzosi, Shifted symplectic structures, Publ. Math. IHES. 117 (2013), 271-328. 1.8

[29] M. Romagny, Group actions on stacks and applications, Michigan Math. J. 53 (2005), no. $1,209-236.4 .3$

[30] J. Shen, The Euler characteristics of generalized Kummer schemes, Math. Z. 281 (2015), no. 3-4, 1183-1189. 1.1, 1.3, 1.4 3.1, 3.6

[31] Y. Toda, Generating functions of stable pair invariants via wall-crossings in derived categories, New developments in algebraic geometry, integrable systems and mirror symmetry (RIMS, Kyoto, 2008), 389-434, Adv. Stud. Pure Math., 59, Math. Soc. Japan, Tokyo, 2010. 4.10, 4.11, 4.11

[32] Y. Toda, Curve counting theories via stable objects I: DT/PT correspondence, J. Amer. Math. Soc. 23 (2010), 1119-1157. 1.5

[33] Y. Toda, Multiple cover formula of generalized DT invariants II: Jacobian localizations, arXiv:1108.4993. 4.10

[34] Y. Toda, Stability conditions and curve counting invariants on Calabi-Yau 3-folds, Kyoto J. Math. 52 (2012), no. 1, 1-50. 1.7, 9, 5.2, 5.3

[35] Y. Toda, Hall algebras in the derived category and higher rank DT invariants, arXiv: $1601.07519,1.5,1.8,4.9,4.9,4.10,4.11,4.11,4.11,4.11,5.5,6.4$

Mit, Department of Mathematics

E-mail address: georgo@mit.edu

ETH ZÜRICh, Department of Mathematics

E-mail address: junliang.shen@math.ethz.ch 